\title{
Rhodium-Catalyzed Addition of Arylzinc Reagents to Aryl Alkynyl Ketones: Synthesis of $\beta$, $\beta$-Disubstituted Indanones
}

\author{
Ryo Shintani and Tamio Hayashi* \\ Department of Chemistry, Graduate School of Science, Kyoto University, \\ Sakyo, Kyoto 606-8502, Japan
}

Supporting Information

\section{General}

All air- and moisture-sensitive manipulations were carried out with standard Schlenk techniques under nitrogen or in a glove box under argon.

Dichloromethane and 1,2-dichloroethane were distilled over $\mathrm{CaH}_{2}$ under nitrogen. THF and toluene were distilled over benzophenone ketyl under nitrogen.

$\mathrm{MnO}_{2}$ (Sigma-Aldrich Japan), allyl bromide (TCI), 1,1'bis(diphenylphosphino)ferrocene (Fluka), 1,3-bis(diphenylphosphino)propane (Kanto Chemicals), 1,4-bis(diphenylphosphino)butane (TCI), and triphenylphosphine (Wako Chemicals) were used as received. $\left[\operatorname{RhCl}\left(\mathrm{C}_{2} \mathrm{H}_{4}\right)_{2}\right]_{2}{ }^{1}$, $[\mathrm{RhCl}(\mathrm{cod})]_{2}{ }^{2}$ and ligand $7^{3}$ were synthesized following the literature procedures.

$\alpha$-Arylpropargyl alcohols (precursors of aryl alkynyl ketones) were synthesized from the corresponding terminal alkynes and aromatic aldehydes following the procedure described in the literature. ${ }^{4}$ Arylzinc chlorides were prepared following the literature procedure. ${ }^{5}$

All other chemicals and solvents were purchased from Aldrich, Wako Chemicals, TCI, or Kanto Chemicals and used as received.

\footnotetext{
${ }^{1}$ Cramer, R. Inorg. Synth. 1974, 15, 16.

${ }^{2}$ Giordano, G.; Crabtree, R. H. Inorg. Synth. 1979, 19, 218.

${ }^{3}$ Hayashi, T.; Kanehira, K.; Hagihara, T.; Kumada, M. J. Org. Chem. 1988, 53, 113.

${ }^{4}$ Shintani, R.; Okamoto, K.; Hayashi, T. J. Am. Chem. Soc. 2005, 127, asap.

${ }^{5}$ Shintani, R.; Tokunaga, N.; Doi, H.; Hayashi, T. J. Am. Chem. Soc. 2004, 126, 6240.
} 


\section{Synthesis of Substrates}

The yields have not been optimized.

1-Phenyl-2-heptyn-1-one (1a) (CAS 18998-78-2)<smiles>CCCCC#CC(=O)c1ccccc1</smiles>

$1 \mathrm{a}$

1-Phenyl-2-heptyn-1-ol (790 mg, $4.20 \mathrm{mmol})$ was added to a suspension of $\mathrm{MnO}_{2}$ $(3.65 \mathrm{~g}, 42.0 \mathrm{mmol})$ in dichloromethane $(30 \mathrm{~mL})$ at $0{ }^{\circ} \mathrm{C}$, and the resulting mixture was stirred for $2 \mathrm{~h}$ at $0{ }^{\circ} \mathrm{C}$. After filtered through celite $/ \mathrm{MgSO}_{4}$, the solvent was removed under vacuum. The residue was passed through a pad of silica gel with $\mathrm{Et}_{2} \mathrm{O}$ to afford 1-phenyl-2-heptyn-1-one as a colorless oil (782 mg, $4.20 \mathrm{mmol} ; 100 \%$ yield).

${ }^{1} \mathrm{H} \mathrm{NMR}\left(\mathrm{CDCl}_{3}\right): \delta 8.14\left(\mathrm{dd},{ }^{3} J_{\mathrm{HH}}=8.0 \mathrm{~Hz}\right.$ and $\left.{ }^{4} J_{\mathrm{HH}}=1.0 \mathrm{~Hz}, 2 \mathrm{H}\right), 7.59\left(\mathrm{tt}^{3} J_{\mathrm{HH}}=\right.$ $7.4 \mathrm{~Hz}$ and $\left.{ }^{4} J_{\mathrm{HH}}=1.2 \mathrm{~Hz}, 1 \mathrm{H}\right), 7.47\left(\mathrm{t},{ }^{3} J_{\mathrm{HH}}=8.0 \mathrm{~Hz}, 2 \mathrm{H}\right), 2.51\left(\mathrm{t},{ }^{3} J_{\mathrm{HH}}=7.1 \mathrm{~Hz}, 2 \mathrm{H}\right)$, 1.69-1.64 (m, 2H), 1.55-1.47 (m, 2H), $0.97\left(\mathrm{t},{ }^{3} \mathrm{JHH}_{\mathrm{HH}}=7.5 \mathrm{~Hz}, 3 \mathrm{H}\right) .{ }^{13} \mathrm{C} \mathrm{NMR}\left(\mathrm{CDCl}_{3}\right)$ : $\delta 178.5,137.2,134.0,129.8,128.7,97.0,79.9,30.1,22.3,19.1,13.7$.

1-Phenyl-2-nonyn-1-one (1b) (CAS 56517-81-8)<smiles>O=C(C#CC=Cc1ccccc1)c1ccccc1</smiles>

$1 \mathrm{~b}$

This was synthesized from 1-phenyl-2-nonyn-1-ol, following the procedure for compound 1a. Colorless oil, $89 \%$ yield.

${ }^{1} \mathrm{H} \mathrm{NMR}\left(\mathrm{CDCl}_{3}\right): \delta 8.14\left(\mathrm{dd},{ }^{3} \mathrm{~J}_{\mathrm{HH}}=8.1 \mathrm{~Hz}\right.$ and $\left.{ }^{4} J_{\mathrm{HH}}=0.7 \mathrm{~Hz}, 2 \mathrm{H}\right), 7.59\left(\mathrm{t}^{3} \mathrm{~J}_{\mathrm{HH}}=\right.$ $7.3 \mathrm{~Hz}, 1 \mathrm{H}), 7.47\left(\mathrm{t},{ }^{3} J_{\mathrm{HH}}=7.8 \mathrm{~Hz}, 2 \mathrm{H}\right), 2.50\left(\mathrm{t},{ }^{3} J_{\mathrm{HH}}=7.1 \mathrm{~Hz}, 2 \mathrm{H}\right), 1.68$ (quint, ${ }^{3} J_{\mathrm{HH}}=$ $7.2 \mathrm{~Hz}, 2 \mathrm{H}), 1.51-1.45(\mathrm{~m}, 2 \mathrm{H}), 1.36-1.31(\mathrm{~m}, 4 \mathrm{H}), 0.91\left(\mathrm{t},{ }^{3} \mathrm{~J}_{\mathrm{HH}}=6.9 \mathrm{~Hz}, 3 \mathrm{H}\right) .{ }^{13} \mathrm{C} \mathrm{NMR}$ $\left(\mathrm{CDCl}_{3}\right): \delta 178.5,137.2,134.0,129.8,128.7,97.1,79.9,31.4,28.9,28.0,22.7,19.4,14.2$.

1-Phenyl-5-methyl-2-hexyn-1-one (1c) (CAS 65236-44-4)<smiles>CC(C)(C)C#CC(=O)c1ccccc1</smiles>

$1 \mathrm{c}$

This was synthesized from 1-phenyl-5-methyl-2-hexyn-1-ol, following the procedure for compound 1a. Colorless oil, $100 \%$ yield.

${ }^{1} \mathrm{H} \mathrm{NMR}\left(\mathrm{CDCl}_{3}\right): \delta 8.14\left(\mathrm{dd},{ }^{3} J_{\mathrm{HH}}=8.2 \mathrm{~Hz}\right.$ and $\left.{ }^{4} J_{\mathrm{HH}}=1.5 \mathrm{~Hz}, 2 \mathrm{H}\right), 7.59\left(\mathrm{tt}^{3}\right)_{\mathrm{HH}}=$ $7.3 \mathrm{~Hz}$ and $\left.{ }^{4} J_{\mathrm{HH}}=1.2 \mathrm{~Hz}, 1 \mathrm{H}\right), 7.48\left(\mathrm{t},{ }^{3} \mathrm{~J}_{\mathrm{HH}}=7.8 \mathrm{~Hz}, 2 \mathrm{H}\right), 2.41\left(\mathrm{~d},{ }^{3} \mathrm{~J}_{\mathrm{HH}}=6.4 \mathrm{~Hz}, 2 \mathrm{H}\right)$, 2.02 (septet, $\left.{ }^{3} \mathrm{~J}_{\mathrm{HH}}=6.6 \mathrm{~Hz}, 1 \mathrm{H}\right), 1.09\left(\mathrm{~d},{ }^{3} \mathrm{~J}_{\mathrm{HH}}=6.7 \mathrm{~Hz}, 6 \mathrm{H}\right) .{ }^{13} \mathrm{C} \mathrm{NMR}\left(\mathrm{CDCl}_{3}\right): \delta 178.4$, 137.2, 134.0, 129.7, 128.7, 96.0, 80.8, 28.5, 28.0, 22.3.

1-(p-Tolyl)-2-heptyn-1-one (1d) (CAS 38395-06-1) 
<smiles>CCCCOC#CC(=O)c1ccc(C)cc1</smiles>

This was synthesized from 1-(p-tolyl)-2-heptyn-1-ol, following the procedure for compound 1a. Colorless oil, $95 \%$ yield.

${ }^{1} \mathrm{H} \mathrm{NMR}\left(\mathrm{CDCl}_{3}\right): \delta 8.03\left(\mathrm{~d},{ }^{3} J_{\mathrm{HH}}=8.2 \mathrm{~Hz}, 2 \mathrm{H}\right), 7.27\left(\mathrm{~d},{ }^{3} J_{\mathrm{HH}}=8.4 \mathrm{~Hz}, 2 \mathrm{H}\right), 2.50(\mathrm{t}$, $\left.{ }^{3} J_{\mathrm{HH}}=7.1 \mathrm{~Hz}, 2 \mathrm{H}\right), 2.43(\mathrm{~s}, 3 \mathrm{H}), 1.69-1.63(\mathrm{~m}, 2 \mathrm{H}), 1.55-1.47(\mathrm{~m}, 2 \mathrm{H}), 0.97\left(\mathrm{t},{ }^{3} J_{\mathrm{HH}}=7.3\right.$ $\mathrm{Hz}, 3 \mathrm{H}) .{ }^{13} \mathrm{C} \mathrm{NMR}\left(\mathrm{CDCl}_{3}\right): \delta 178.2,145.1,134.9,129.9,129.4,96.5,80.0,30.1,22.3$, $22.0,19.1,13.7$.

\section{1-( $p$-Methoxyphenyl)-2-heptyn-1-one (1e) (CAS 93845-81-9)}

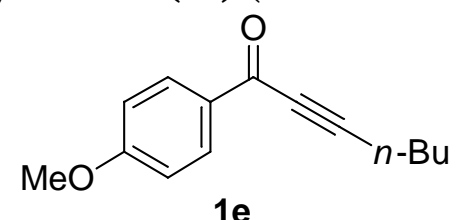

This was synthesized from 1-( $p$-methoxyphenyl)-2-heptyn-1-ol, following the procedure for compound 1a. The crude material was chromatographed on silica gel with $\mathrm{Et}_{2} \mathrm{O} /$ hexane $=1 / 5$. Colorless oil, $70 \%$ yield .

${ }^{1} \mathrm{H} \mathrm{NMR}\left(\mathrm{CDCl}_{3}\right): \delta 8.11-8.10(\mathrm{~m}, 2 \mathrm{H}), 6.95-6.94(\mathrm{~m}, 2 \mathrm{H}), 3.88(\mathrm{~s}, 3 \mathrm{H}), 2.49\left(\mathrm{t},{ }^{3} \mathrm{~J}_{\mathrm{HH}}=\right.$ $7.2 \mathrm{~Hz}, 2 \mathrm{H}), 1.69-1.63(\mathrm{~m}, 2 \mathrm{H}), 1.54-1.47(\mathrm{~m}, 2 \mathrm{H}), 0.96\left(\mathrm{t},{ }^{3} \mathrm{~J}_{\mathrm{HH}}=7.3 \mathrm{~Hz}, 3 \mathrm{H}\right) .{ }^{13} \mathrm{C} \mathrm{NMR}$ $\left(\mathrm{CDCl}_{3}\right): \delta 177.2,164.5,132.1,130.6,113.9,96.1,79.9,55.8,30.1,22.3,19.1,13.7$.

\section{1-(p-Fluorophenyl)-2-heptyn-1-one (1f)}<smiles>CCCCOC#CC(=O)c1ccc(F)cc1</smiles>

This was synthesized from 1-( $p$-fluorophenyl)-2-heptyn-1-ol, following the procedure for compound 1a. Colorless oil, $89 \%$ yield.

${ }^{1} \mathrm{H}$ NMR $\left(\mathrm{CDCl}_{3}\right): \delta 8.18-8.14(\mathrm{~m}, 2 \mathrm{H}), 7.17-7.12(\mathrm{~m}, 2 \mathrm{H}), 2.50\left(\mathrm{t},{ }^{3} J_{\mathrm{HH}}=7.2 \mathrm{~Hz}, 2 \mathrm{H}\right)$, 1.69-1.64 (m, 2H), 1.54-1.47 (m, 2H), $0.97\left(\mathrm{t},{ }^{3} \mathrm{~J}_{\mathrm{HH}}=7.3 \mathrm{~Hz}, 3 \mathrm{H}\right) .{ }^{13} \mathrm{C} \mathrm{NMR}\left(\mathrm{CDCl}_{3}\right)$ : $\delta 176.8,166.5\left(\mathrm{~d},{ }^{1} J_{\mathrm{CF}}=255.8 \mathrm{~Hz}\right), 133.7\left(\mathrm{~d},{ }^{4} J_{\mathrm{CF}}=3.1 \mathrm{~Hz}\right), 132.4\left(\mathrm{~d},{ }^{3} J_{\mathrm{CF}}=9.3 \mathrm{~Hz}\right), 115.9$ $\left(\mathrm{d},{ }^{2} \mathrm{~J}_{\mathrm{HH}}=22.2 \mathrm{~Hz}\right), 97.3,79.6,30.0,22.3,19.1,13.7$. Anal. Calcd for $\mathrm{C}_{13} \mathrm{H}_{13} \mathrm{FO}: \mathrm{C}$, 76.45; H, 6.42. Found: C, 76.36; H, 6.41.

\section{1-(o-Tolyl)-2-heptyn-1-one (1g)}<smiles>CCCCCC#CC(=O)c1ccccc1C</smiles>

19

This was synthesized from 1-(o-tolyl)-2-heptyn-1-ol, following the procedure for compound 1a. The crude material was chromatographed on silica gel with $\mathrm{Et}_{2} \mathrm{O} /$ hexane $=1 / 5$. Colorless oil, $65 \%$ yield .

${ }^{1} \mathrm{H} \mathrm{NMR}\left(\mathrm{CDCl}_{3}\right): \delta 8.18\left(\mathrm{~d},{ }^{3} \mathrm{~J}_{\mathrm{HH}}=7.8 \mathrm{~Hz}, 1 \mathrm{H}\right), 7.42\left(\mathrm{t},{ }^{3} \mathrm{~J}_{\mathrm{HH}}=7.4 \mathrm{~Hz}, 1 \mathrm{H}\right), 7.31(\mathrm{t}$, $\left.{ }^{3} J_{\mathrm{HH}}=7.8 \mathrm{~Hz}, 1 \mathrm{H}\right), 7.24\left(\mathrm{~d},{ }^{3} J_{\mathrm{HH}}=7.5 \mathrm{~Hz}, 1 \mathrm{H}\right), 2.62(\mathrm{~s}, 3 \mathrm{H}), 2.47\left(\mathrm{t},{ }^{3} J_{\mathrm{HH}}=7.1 \mathrm{~Hz}, 2 \mathrm{H}\right)$, 
1.67-1.62 (m, 2H), 1.53-1.46 (m, 2H), $0.96\left(\mathrm{t},{ }^{3} \mathrm{~J}_{\mathrm{HH}}=7.4 \mathrm{~Hz}, 3 \mathrm{H}\right) .{ }^{13} \mathrm{C} \mathrm{NMR}\left(\mathrm{CDCl}_{3}\right)$ : $\delta 180.2,140.5,136.1,133.4,132.8,132.2,125.9,95.6,81.4,30.1,22.3,22.1,19.1,13.7$. Anal. Calcd for $\mathrm{C}_{14} \mathrm{H}_{16} \mathrm{O}: \mathrm{C}, 83.96 ; \mathrm{H}, 8.05$. Found: $\mathrm{C}, 84.04 ; \mathrm{H}, 8.09$. 


\section{Catalytic Reactions}

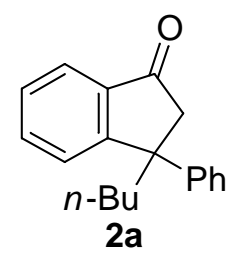

Procedure for Table 1, entry 1.

1-Phenyl-2-heptyn-1-one $1 \mathrm{a}(37.3 \mathrm{mg}, 0.20 \mathrm{mmol})$ was added to a solution of $\left[\mathrm{RhCl}\left(\mathrm{C}_{2} \mathrm{H}_{4}\right)_{2}\right]_{2}(3.9 \mathrm{mg}, 20 \mu \mathrm{mol} \mathrm{Rh})$ and dppf $(12.2 \mathrm{mg}, 22 \mu \mathrm{mol})$ in 1,2dichloroethane $(10 \mathrm{~mL})$. PhZnCl $(625 \mu \mathrm{L}, 0.30 \mathrm{mmol} ; 0.48 \mathrm{M}$ in THF) was then added to it and the resulting solution was stirred for $7 \mathrm{~h}$ at $25^{\circ} \mathrm{C}$. The reaction was quenched with water $(30 \mu \mathrm{L})$ and passed through a pad of silica gel with $\mathrm{Et}_{2} \mathrm{O}$. The solvent was removed under vacuum and the residue was purified by silica gel preparative TLC with $\mathrm{Et}_{2} \mathrm{O} /$ hexane $=1 / 4.5$ to afford indanone $2 \mathrm{a}$ as a pale yellow oil (42.1 mg, $0.16 \mathrm{mmol} ; 80 \%$ yield).

${ }^{1} \mathrm{H} \mathrm{NMR}\left(\mathrm{CDCl}_{3}\right): \delta 7.78\left(\mathrm{~d},{ }^{3} \mathrm{~J}_{\mathrm{HH}}=7.7 \mathrm{~Hz}, 1 \mathrm{H}\right), 7.60\left(\mathrm{t},{ }^{3} \mathrm{~J}_{\mathrm{HH}}=7.4 \mathrm{~Hz}, 1 \mathrm{H}\right), 7.42(\mathrm{t}$, $\left.{ }^{3} J_{\mathrm{HH}}=7.4 \mathrm{~Hz}, 1 \mathrm{H}\right), 7.33\left(\mathrm{~d},{ }^{3} J_{\mathrm{HH}}=7.8 \mathrm{~Hz}, 1 \mathrm{H}\right), 7.31-7.27(\mathrm{~m}, 2 \mathrm{H}), 7.23-7.18(\mathrm{~m}, 3 \mathrm{H})$, $2.96\left(\mathrm{~d},{ }^{2} J_{\mathrm{HH}}=19.1 \mathrm{~Hz}, 1 \mathrm{H}\right), 2.92\left(\mathrm{~d},{ }^{2} J_{\mathrm{HH}}=19.0 \mathrm{~Hz}, 1 \mathrm{H}\right), 2.27\left(\mathrm{ddd},{ }^{2} J_{\mathrm{HH}}=13.6 \mathrm{~Hz}\right.$ and ${ }^{3} J_{\mathrm{HH}}=12.5$ and $\left.4.3 \mathrm{~Hz}, 1 \mathrm{H}\right), 2.15\left(\mathrm{ddd},{ }^{2} J_{\mathrm{HH}}=13.4 \mathrm{~Hz}\right.$ and ${ }^{3} J_{\mathrm{HH}}=12.3$ and $\left.4.2 \mathrm{~Hz}, 1 \mathrm{H}\right)$, $1.34-1.26(\mathrm{~m}, 2 \mathrm{H}), 1.25-1.16(\mathrm{~m}, 1 \mathrm{H}), 0.92-0.85(\mathrm{~m}, 1 \mathrm{H}), 0.84\left(\mathrm{t},{ }^{3} J_{\mathrm{HH}}=7.3 \mathrm{~Hz}, 3 \mathrm{H}\right) .{ }^{13} \mathrm{C}$ NMR $\left(\mathrm{CDCl}_{3}\right): \delta 206.0,161.0,147.2,137.0,135.1,128.7,127.9,126.7,126.5,126.4,123.6$, 53.1, 50.1, 40.0, 27.3, 23.4, 14.1. Anal. Calcd for $\mathrm{C}_{19} \mathrm{H}_{20} \mathrm{O}$ : $\mathrm{C}, 86.32 ; \mathrm{H}, 7.63$. Found: $\mathrm{C}$, $86.61 ; \mathrm{H}, 7.66$.

\section{General Procedure for Table 2.}

Aryl alkynyl ketone $\mathbf{1}(0.20 \mathrm{mmol})$ was added to a solution of $\left[\mathrm{RhCl}\left(\mathrm{C}_{2} \mathrm{H}_{4}\right)_{2}\right]_{2}(3.9$ $\mathrm{mg}, 20 \mu \mathrm{mol} \mathrm{Rh})$ and dppf (12.2 mg, $22 \mu \mathrm{mol})$ in 1,2-dichloroethane (10-15 mL). $\mathrm{PhZnCl}(625 \mu \mathrm{L}, 0.30 \mathrm{mmol} ; 0.48 \mathrm{M}$ in THF) was then added to it and the resulting solution was stirred for $7-19 \mathrm{~h}$ at $25^{\circ} \mathrm{C}$. The reaction was quenched with water (30 $\mu \mathrm{L}$ ) and passed through a pad of silica gel with $\mathrm{Et}_{2} \mathrm{O}$. The solvent was removed under vacuum and the residue was purified by silica gel preparative TLC with $\mathrm{Et}_{2} \mathrm{O} /$ hexane to afford indanone 2 .

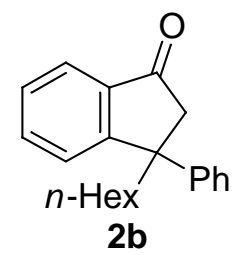

Entry 2. Pale yellow oil. $84 \%$ yield.

${ }^{1} \mathrm{H} \mathrm{NMR}\left(\mathrm{CDCl}_{3}\right): \delta 7.78\left(\mathrm{~d},{ }^{3} \mathrm{~J}_{\mathrm{HH}}=7.7 \mathrm{~Hz}, 1 \mathrm{H}\right), 7.60\left(\mathrm{t},{ }^{3} \mathrm{~J}_{\mathrm{HH}}=7.4 \mathrm{~Hz}, 1 \mathrm{H}\right), 7.42(\mathrm{t}$, $\left.{ }^{3} J_{\mathrm{HH}}=7.7 \mathrm{~Hz}, 1 \mathrm{H}\right), 7.34-7.27(\mathrm{~m}, 3 \mathrm{H}), 7.23-7.18(\mathrm{~m}, 3 \mathrm{H}), 2.96\left(\mathrm{~d},{ }^{2} J_{\mathrm{HH}}=19.1 \mathrm{~Hz}, 1 \mathrm{H}\right)$, $2.92\left(\mathrm{~d},{ }^{2} J_{\mathrm{HH}}=19.0 \mathrm{~Hz}, 1 \mathrm{H}\right), 2.27\left(\mathrm{ddd},{ }^{2} J_{\mathrm{HH}}=13.5 \mathrm{~Hz}\right.$ and ${ }^{3} J_{\mathrm{HH}}=12.0$ and $\left.4.2 \mathrm{~Hz}, 1 \mathrm{H}\right)$, $2.14\left(\mathrm{ddd},{ }^{2} J_{\mathrm{HH}}=13.5 \mathrm{~Hz}\right.$ and ${ }^{3} J_{\mathrm{HH}}=12.3$ and $\left.4.6 \mathrm{~Hz}, 1 \mathrm{H}\right), 1.31-1.17(\mathrm{~m}, 7 \mathrm{H}), 0.92-0.85$ $(\mathrm{m}, 1 \mathrm{H}), 0.84\left(\mathrm{t},{ }^{3} \mathrm{~J}_{\mathrm{HH}}=7.1 \mathrm{~Hz}, 3 \mathrm{H}\right) .{ }^{13} \mathrm{C} \mathrm{NMR}\left(\mathrm{CDCl}_{3}\right): \delta 206.1,161.1,147.2,137.0$, $135.1,128.7,128.0,126.7,126.6,126.4,123.6,53.1,50.2,40.3,31.8,30.0,25.1,22.8,14.2$. Anal. Calcd for $\mathrm{C}_{21} \mathrm{H}_{24} \mathrm{O}: \mathrm{C}, 86.26 ; \mathrm{H}, 8.27$. Found: $\mathrm{C}, 86.08 ; \mathrm{H}, 8.36$. 
Entry 3. Pale yellow oil. $75 \%$ yield.

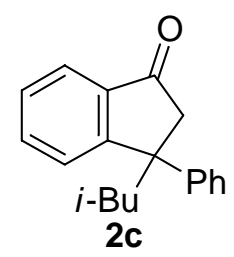

${ }^{1} \mathrm{H} \mathrm{NMR}\left(\mathrm{CDCl}_{3}\right): \delta 7.78\left(\mathrm{~d},{ }^{3} \mathrm{~J}_{\mathrm{HH}}=7.8 \mathrm{~Hz}, 1 \mathrm{H}\right), 7.60\left(\mathrm{t},{ }^{3} J_{\mathrm{HH}}=7.8 \mathrm{~Hz}, 1 \mathrm{H}\right), 7.43-7.40$ $(\mathrm{m}, 2 \mathrm{H}), 7.29-7.23(\mathrm{~m}, 4 \mathrm{H}), 7.18\left(\mathrm{t}^{3} J_{\mathrm{HH}}=6.9 \mathrm{~Hz}, 1 \mathrm{H}\right), 3.04\left(\mathrm{~d},{ }^{2} J_{\mathrm{HH}}=19.1 \mathrm{~Hz}, 1 \mathrm{H}\right), 2.98$ $\left(\mathrm{d},{ }^{2} J_{\mathrm{HH}}=18.9 \mathrm{~Hz}, 1 \mathrm{H}\right), 2.33\left(\mathrm{dd},{ }^{2} J_{\mathrm{HH}}=14.1 \mathrm{~Hz}\right.$ and $\left.{ }^{3} J_{\mathrm{HH}}=4.6 \mathrm{~Hz}, 1 \mathrm{H}\right), 2.10\left(\mathrm{dd},{ }^{2} J_{\mathrm{HH}}\right.$ $=14.0 \mathrm{~Hz}$ and $\left.{ }^{3} J_{\mathrm{HH}}=6.6 \mathrm{~Hz}, 1 \mathrm{H}\right), 1.51-1.45(\mathrm{~m}, 1 \mathrm{H}), 0.89\left(\mathrm{~d},{ }^{3} J_{\mathrm{HH}}=6.7 \mathrm{~Hz}, 3 \mathrm{H}\right), 0.59$ $\left(\mathrm{d},{ }^{3} \mathrm{~J}_{\mathrm{HH}}=6.6 \mathrm{~Hz}, 3 \mathrm{H}\right) .{ }^{13} \mathrm{C} \mathrm{NMR}\left(\mathrm{CDCl}_{3}\right): \delta 206.1,161.0,147.7,137.0,134.9,128.7$, 128.0, 126.8, 126.6, 126.5, 123.6, 53.2, 50.2, 48.9, 25.5, 25.4, 24.4. Anal. Calcd for $\mathrm{C}_{19} \mathrm{H}_{20} \mathrm{O}: \mathrm{C}, 86.32 ; \mathrm{H}, 7.63$. Found: $\mathrm{C}, 86.58 ; \mathrm{H}, 7.84$.

Entry 4. Pale yellow oil. $74 \%$ yield.

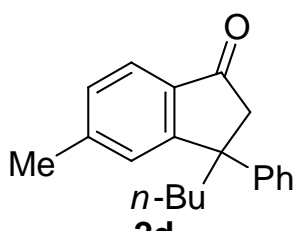

2d

${ }^{1} \mathrm{H}$ NMR $\left(\mathrm{CDCl}_{3}\right): \delta 7.67\left(\mathrm{~d},{ }^{3} J_{\mathrm{HH}}=7.9 \mathrm{~Hz}, 1 \mathrm{H}\right), 7.31-7.27(\mathrm{~m}, 2 \mathrm{H}), 7.24-7.18(\mathrm{~m}$, $4 \mathrm{H}), 7.09(\mathrm{~s}, 1 \mathrm{H}), 2.94\left(\mathrm{~d},{ }^{2} J_{\mathrm{HH}}=18.9 \mathrm{~Hz}, 1 \mathrm{H}\right), 2.89\left(\mathrm{~d},{ }^{2} J_{\mathrm{HH}}=18.9 \mathrm{~Hz}, 1 \mathrm{H}\right), 2.41(\mathrm{~s}, 3 \mathrm{H})$, $2.28\left(\mathrm{td}, J_{\mathrm{HH}}=13.3 \mathrm{~Hz}\right.$ and $\left.{ }^{3} J_{\mathrm{HH}}=4.1 \mathrm{~Hz}, 1 \mathrm{H}\right), 2.12\left(\mathrm{td}, J_{\mathrm{HH}}=13.3 \mathrm{~Hz}\right.$ and ${ }^{3} J_{\mathrm{HH}}=4.4$ $\mathrm{Hz}, 1 \mathrm{H}), 1.36-1.27(\mathrm{~m}, 2 \mathrm{H}), 1.25-1.15(\mathrm{~m}, 1 \mathrm{H}), 0.94-0.84(\mathrm{~m}, 1 \mathrm{H}), 0.85\left(\mathrm{t},{ }^{3} J_{\mathrm{HH}}=7.2 \mathrm{~Hz}\right.$, $3 \mathrm{H}) .{ }^{13} \mathrm{C} \mathrm{NMR}\left(\mathrm{CDCl}_{3}\right): \delta$ 205.6, 161.7, 147.4, 146.3, 134.8, 129.3, 128.7, 126.7, 126.5, 126.4, 123.4, 53.3, 49.8, 39.8, 27.3, 23.4, 22.5, 14.1. Anal. Calcd for $\mathrm{C}_{20} \mathrm{H}_{22} \mathrm{O}: \mathrm{C}, 86.29$; H, 7.97. Found: C, 86.04; H, 8.03.

Entry 5. Pale yellow oil. $63 \%$ yield.

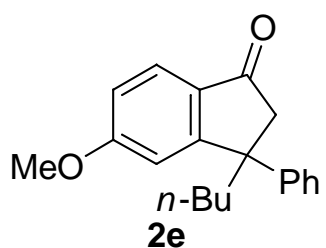

${ }^{1} \mathrm{H} \mathrm{NMR}\left(\mathrm{CDCl}_{3}\right): \delta 7.72\left(\mathrm{~d},{ }^{3} \mathrm{~J}_{\mathrm{HH}}=8.5 \mathrm{~Hz}, 1 \mathrm{H}\right), 7.31-7.27(\mathrm{~m}, 2 \mathrm{H}), 7.24-7.18(\mathrm{~m}$, $3 \mathrm{H}), 6.94\left(\mathrm{dd},{ }^{3} J_{\mathrm{HH}}=8.5 \mathrm{~Hz}\right.$ and $\left.{ }^{4} J_{\mathrm{HH}}=2.2 \mathrm{~Hz}, 1 \mathrm{H}\right), 6.69\left(\mathrm{~d},{ }^{4} J_{\mathrm{HH}}=2.2 \mathrm{~Hz}, 1 \mathrm{H}\right), 3.83(\mathrm{~s}$, $3 \mathrm{H}), 2.93\left(\mathrm{~d},{ }^{2} J_{\mathrm{HH}}=18.9 \mathrm{~Hz}, 1 \mathrm{H}\right), 2.88\left(\mathrm{~d},{ }^{2} J_{\mathrm{HH}}=18.8 \mathrm{~Hz}, 1 \mathrm{H}\right), 2.28\left(\mathrm{ddd},{ }^{2} J_{\mathrm{HH}}=13.4\right.$ $\mathrm{Hz}$ and ${ }^{3} J_{\mathrm{HH}}=12.3$ and $\left.4.2 \mathrm{~Hz}, 1 \mathrm{H}\right), 2.11\left(\mathrm{ddd},{ }^{2} J_{\mathrm{HH}}=13.4 \mathrm{~Hz}\right.$ and ${ }^{3} J_{\mathrm{HH}}=12.4$ and 4.4 $\mathrm{Hz}, 1 \mathrm{H}), 1.38-1.27(\mathrm{~m}, 2 \mathrm{H}), 1.25-1.16(\mathrm{~m}, 1 \mathrm{H}), 0.94-0.86(\mathrm{~m}, 1 \mathrm{H}), 0.85\left(\mathrm{t},{ }^{3} J_{\mathrm{HH}}=7.2 \mathrm{~Hz}\right.$, $3 \mathrm{H}) .{ }^{13} \mathrm{C}$ NMR $\left(\mathrm{CDCl}_{3}\right): \delta 204.2,165.7,164.1,147.3,130.5,128.7,126.6,126.5,125.3$, 115.6, 109.7, 55.9, 53.3, 49.9, 39.6, 27.2, 23.4, 14.1. Anal. Calcd for $\mathrm{C}_{20} \mathrm{H}_{22} \mathrm{O}_{2}$ : C, 81.60; H, 7.53. Found: C, 81.43; H, 7.49. 
Entry 6. Pale yellow oil. $68 \%$ yield.

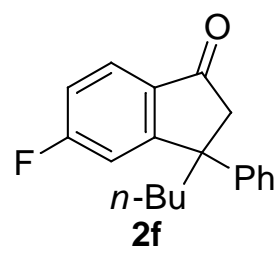

${ }^{1} \mathrm{H} \mathrm{NMR}\left(\mathrm{CDCl}_{3}\right): \delta 7.78\left(\mathrm{dd},{ }^{3} J_{\mathrm{HH}}=8.4 \mathrm{~Hz}\right.$ and $\left.{ }^{4} J_{\mathrm{HF}}=5.4 \mathrm{~Hz}, 1 \mathrm{H}\right), 7.32-7.29(\mathrm{~m}$, $2 \mathrm{H}), 7.23-7.20(\mathrm{~m}, 3 \mathrm{H}), 7.11\left(\mathrm{td},{ }^{3} J=8.5 \mathrm{~Hz}\right.$ and $\left.{ }^{4} J_{\mathrm{HH}}=2.2 \mathrm{~Hz}, 1 \mathrm{H}\right), 6.96\left(\mathrm{dd},{ }^{3} J_{\mathrm{HF}}=8.8\right.$ $\mathrm{Hz}$ and $\left.{ }^{4} J_{\mathrm{HH}}=2.2 \mathrm{~Hz}, 1 \mathrm{H}\right), 2.97\left(\mathrm{~d},{ }^{2} J_{\mathrm{HH}}=19.2 \mathrm{~Hz}, 1 \mathrm{H}\right), 2.93\left(\mathrm{~d},{ }^{2} J_{\mathrm{HH}}=19.2 \mathrm{~Hz}, 1 \mathrm{H}\right)$, $2.28\left(\mathrm{ddd},{ }^{2} J_{\mathrm{HH}}=13.7 \mathrm{~Hz}\right.$ and ${ }^{3} J_{\mathrm{HH}}=12.2$ and $\left.4.2 \mathrm{~Hz}, 1 \mathrm{H}\right), 2.12\left(\mathrm{ddd},{ }^{2} J_{\mathrm{HH}}=13.6 \mathrm{~Hz}\right.$ and ${ }^{3} J_{\mathrm{HH}}=12.3$ and $\left.4.4 \mathrm{~Hz}, 1 \mathrm{H}\right), 1.39-1.26(\mathrm{~m}, 2 \mathrm{H}), 1.25-1.16(\mathrm{~m}, 1 \mathrm{H}), 0.94-0.85(\mathrm{~m}$, $1 \mathrm{H}), 0.86\left(\mathrm{t},{ }^{3} J_{\mathrm{HH}}=7.3 \mathrm{~Hz}, 3 \mathrm{H}\right) .{ }^{13} \mathrm{C} \mathrm{NMR}\left(\mathrm{CDCl}_{3}\right): \delta 204.0,167.5\left(\mathrm{~d},{ }^{1} J_{\mathrm{CF}}=256.3 \mathrm{~Hz}\right)$, $164.2\left(\mathrm{~d},{ }^{3} J_{\mathrm{CF}}=8.8 \mathrm{~Hz}\right), 146.5,133.4\left(\mathrm{~d},{ }^{4} J_{\mathrm{CF}}=2.1 \mathrm{~Hz}\right), 128.9,126.8,126.5,125.9\left(\mathrm{~d},{ }^{3} J_{\mathrm{CF}}\right.$ $=10.9 \mathrm{~Hz}), 116.4\left(\mathrm{~d},{ }^{2} J_{\mathrm{CF}}=23.7 \mathrm{~Hz}\right), 112.9\left(\mathrm{~d},{ }^{1} J_{\mathrm{CF}}=22.2 \mathrm{~Hz}\right), 53.2,50.0\left(\mathrm{~d},{ }^{4} J_{\mathrm{CF}}=2.1\right.$ $\mathrm{Hz}), 39.7,27.2$, 23.3, 14.1. HRMS (ESI) calcd for $\mathrm{C}_{19} \mathrm{H}_{19} \mathrm{FONa}\left(\mathrm{M}+\mathrm{Na}^{+}\right)$305.1312, found 305.1309 .

Entry 7. Pale yellow oil. 77\% yield.

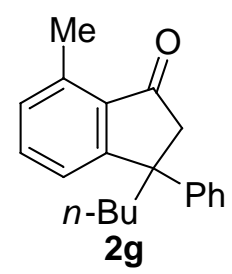

${ }^{1} \mathrm{H} \mathrm{NMR}\left(\mathrm{CDCl}_{3}\right): \delta 7.43\left(\mathrm{t},{ }^{3} J_{\mathrm{HH}}=7.4 \mathrm{~Hz}, 1 \mathrm{H}\right), 7.30-7.27(\mathrm{~m}, 2 \mathrm{H}), 7.23-7.17(\mathrm{~m}, 3 \mathrm{H})$, $7.13\left(\mathrm{t},{ }^{3} J_{\mathrm{HH}}=8.3 \mathrm{~Hz}, 2 \mathrm{H}\right), 2.94\left(\mathrm{~d},{ }^{2} J_{\mathrm{HH}}=19.1 \mathrm{~Hz}, 1 \mathrm{H}\right), 2.90\left(\mathrm{~d},{ }^{2} J_{\mathrm{HH}}=19.6 \mathrm{~Hz}, 1 \mathrm{H}\right)$, $2.69(\mathrm{~s}, 3 \mathrm{H}), 2.27\left(\mathrm{ddd},{ }^{2} J_{\mathrm{HH}}=13.4 \mathrm{~Hz}\right.$ and ${ }^{3} J_{\mathrm{HH}}=12.3$ and $\left.4.2 \mathrm{~Hz}, 1 \mathrm{H}\right), 2.12\left(\mathrm{ddd},{ }^{2} J_{\mathrm{HH}}\right.$ $=13.4 \mathrm{~Hz}$ and ${ }^{3} J_{\mathrm{HH}}=12.3$ and $\left.4.4 \mathrm{~Hz}, 1 \mathrm{H}\right), 1.38-1.28(\mathrm{~m}, 2 \mathrm{H}), 1.24-1.17(\mathrm{~m}, 1 \mathrm{H}), 0.95-$ $0.88(\mathrm{~m}, 1 \mathrm{H}), 0.85\left(\mathrm{t},{ }^{3} \mathrm{~J}_{\mathrm{HH}}=7.3 \mathrm{~Hz}, 3 \mathrm{H}\right) .{ }^{13} \mathrm{C} \mathrm{NMR}\left(\mathrm{CDCl}_{3}\right): \delta 207.0,161.9,147.6,138.6$, 134.4, 134.3, 129.7, 128.7, 126.7, 126.4, 123.7, 53.5, 49.3, 40.0, 27.3, 23.4, 18.7, 14.1. Anal. Calcd for $\mathrm{C}_{20} \mathrm{H}_{22} \mathrm{O}: \mathrm{C}, 86.29 ; \mathrm{H}, 7.97$. Found: $\mathrm{C}, 86.22 ; \mathrm{H}, 8.05$.

\section{General Procedure for Table 3.}

1-Phenyl-2-heptyn-1-one $1 \mathrm{1a}(37.3 \mathrm{mg}, 0.20 \mathrm{mmol})$ was added to a solution of $\left[\mathrm{RhCl}\left(\mathrm{C}_{2} \mathrm{H}_{4}\right)_{2}\right]_{2}(3.9 \mathrm{mg}, 20 \mu \mathrm{mol} \mathrm{Rh})$ and dppf $(12.2 \mathrm{mg}, 22 \mu \mathrm{mol})$ in $1,2-$ dichloroethane $(10-15 \mathrm{~mL})$. ArZnCl $(0.30 \mathrm{mmol} ; 0.48-0.79 \mathrm{M}$ in THF) was then added to it and the resulting solution was stirred for $7-17 \mathrm{~h}$ at $25{ }^{\circ} \mathrm{C}$. The reaction was quenched with water $(30 \mu \mathrm{L})$ and passed through a pad of silica gel with $\mathrm{Et}_{2} \mathrm{O}$. The solvent was removed under vacuum and the residue was purified by silica gel preparative TLC with $\mathrm{Et}_{2} \mathrm{O} /$ hexane to afford indanone 2.

Entry 2. Pale yellow oil. $81 \%$ yield.

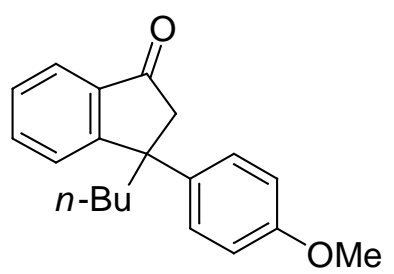

$2 \mathrm{~h}$ 
${ }^{1} \mathrm{H}$ NMR $\left(\mathrm{CDCl}_{3}\right): \delta 7.77\left(\mathrm{~d},{ }^{3} J_{\mathrm{HH}}=7.5 \mathrm{~Hz}, 1 \mathrm{H}\right), 7.59\left(\mathrm{t},{ }^{3} J_{\mathrm{HH}}=7.3 \mathrm{~Hz}, 1 \mathrm{H}\right), 7.40(\mathrm{t}$, $\left.{ }^{3} J_{\mathrm{HH}}=7.3 \mathrm{~Hz}, 1 \mathrm{H}\right), 7.32\left(\mathrm{~d},{ }^{3} J_{\mathrm{HH}}=7.7 \mathrm{~Hz}, 1 \mathrm{H}\right), 7.13\left(\mathrm{~d},{ }^{3} J_{\mathrm{HH}}=8.7 \mathrm{~Hz}, 2 \mathrm{H}\right), 6.82\left(\mathrm{~d},{ }^{3} J_{\mathrm{HH}}\right.$ $=8.6 \mathrm{~Hz}, 2 \mathrm{H}), 3.77(\mathrm{~s}, 3 \mathrm{H}), 2.94\left(\mathrm{~d},{ }^{2} J_{\mathrm{HH}}=19.0 \mathrm{~Hz}, 1 \mathrm{H}\right), 2.88\left(\mathrm{~d},{ }^{2} J_{\mathrm{HH}}=18.9 \mathrm{~Hz}, 1 \mathrm{H}\right)$, $2.24\left(\mathrm{td}, J_{\mathrm{HH}}=13.3 \mathrm{~Hz}\right.$ and $\left.{ }^{3} J_{\mathrm{HH}}=4.1 \mathrm{~Hz}, 1 \mathrm{H}\right), 2.12\left(\mathrm{td}, J_{\mathrm{HH}}=13.3 \mathrm{~Hz}\right.$ and ${ }^{3} J_{\mathrm{HH}}=4.2$ $\mathrm{Hz}, 1 \mathrm{H}), 1.36-1.25(\mathrm{~m}, 2 \mathrm{H}), 1.23-1.15(\mathrm{~m}, 1 \mathrm{H}), 0.92-0.84(\mathrm{~m}, 1 \mathrm{H}), 0.84\left(\mathrm{t},{ }^{3} J_{\mathrm{HH}}=7.3 \mathrm{~Hz}\right.$, $3 \mathrm{H}) .{ }^{13} \mathrm{C}$ NMR $\left(\mathrm{CDCl}_{3}\right): \delta 206.2,161.3,158.1,139.3,136.9,135.1,127.9,127.7,126.3$, 123.5, 114.0, 55.4, 53.2, 49.5, 40.2, 27.3, 23.4, 14.1. Anal. Calcd for $\mathrm{C}_{20} \mathrm{H}_{22} \mathrm{O}_{2}$ : C, 81.60; $\mathrm{H}, 7.53$. Found: $\mathrm{C}, 81.35 ; \mathrm{H}, 7.53$.

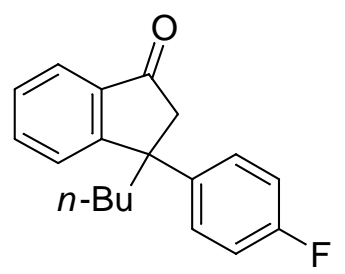

Entry 3. Pale yellow solid. $72 \%$ yield.

2i

${ }^{1} \mathrm{H} \mathrm{NMR}\left(\mathrm{CDCl}_{3}\right): \delta 7.78\left(\mathrm{~d},{ }^{3} J_{\mathrm{HH}}=7.5 \mathrm{~Hz}, 1 \mathrm{H}\right), 7.61\left(\mathrm{t},{ }^{3} J_{\mathrm{HH}}=7.8 \mathrm{~Hz}, 1 \mathrm{H}\right), 7.43(\mathrm{t}$, $\left.{ }^{3} J_{\mathrm{HH}}=7.7 \mathrm{~Hz}, 1 \mathrm{H}\right), 7.31\left(\mathrm{~d},{ }^{3} J_{\mathrm{HH}}=7.8 \mathrm{~Hz}, 1 \mathrm{H}\right), 7.17\left(\mathrm{dd},{ }^{3} J_{\mathrm{HH}}=8.9 \mathrm{~Hz}\right.$ and ${ }^{4} J_{\mathrm{HF}}=5.2$ $\mathrm{Hz}, 2 \mathrm{H}), 6.97\left(\mathrm{t},{ }^{3} \mathrm{~J}=8.6 \mathrm{~Hz}, 2 \mathrm{H}\right), 2.95\left(\mathrm{~d},{ }^{2} J_{\mathrm{HH}}=19.1 \mathrm{~Hz}, 1 \mathrm{H}\right), 2.86\left(\mathrm{~d},{ }^{2} J_{\mathrm{HH}}=19.0 \mathrm{~Hz}\right.$, $1 \mathrm{H}), 2.24\left(\mathrm{ddd},{ }^{2} J_{\mathrm{HH}}=13.4 \mathrm{~Hz}\right.$ and ${ }^{3} J_{\mathrm{HH}}=12.3$ and $\left.4.4 \mathrm{~Hz}, 1 \mathrm{H}\right), 2.13\left(\mathrm{ddd},{ }^{2} J_{\mathrm{HH}}=13.4\right.$ $\mathrm{Hz}$ and ${ }^{3} J_{\mathrm{HH}}=12.3$ and $\left.4.4 \mathrm{~Hz}, 1 \mathrm{H}\right), 1.36-1.25(\mathrm{~m}, 2 \mathrm{H}), 1.22-1.13(\mathrm{~m}, 1 \mathrm{H}), 0.92-0.82(\mathrm{~m}$, $1 \mathrm{H}), 0.84\left(\mathrm{t},{ }^{3} J_{\mathrm{HH}}=7.3 \mathrm{~Hz}, 3 \mathrm{H}\right) .{ }^{13} \mathrm{C}$ NMR $\left(\mathrm{CDCl}_{3}\right): \delta 205.7,161.5\left(\mathrm{~d},{ }^{1} J_{\mathrm{CF}}=245.4 \mathrm{~Hz}\right)$, $160.7,143.1\left(\mathrm{~d},{ }^{4} J_{\mathrm{CF}}=3.0 \mathrm{~Hz}\right), 137.0,135.2,128.3\left(\mathrm{~d},{ }^{3} J_{\mathrm{CF}}=8.3 \mathrm{~Hz}\right), 128.1,126.2,123.7$, $115.5\left(\mathrm{~d},{ }^{2} J_{\mathrm{CF}}=20.7 \mathrm{~Hz}\right), 53.2,49.7,40.2,27.3,23.3$, 14.1. Anal. Calcd for $\mathrm{C}_{19} \mathrm{H}_{19} \mathrm{FO}: \mathrm{C}$, 80.82; H, 6.78. Found: C, 80.63; H, 6.85 .

Entry 4. Pale yellow oil. $74 \%$ yield.

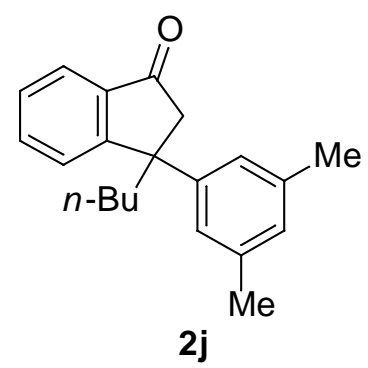

${ }^{1} \mathrm{H} \mathrm{NMR}\left(\mathrm{CDCl}_{3}\right): \delta 7.78\left(\mathrm{~d},{ }^{3} J_{\mathrm{HH}}=7.7 \mathrm{~Hz}, 1 \mathrm{H}\right), 7.60\left(\mathrm{t},{ }^{3} J_{\mathrm{HH}}=7.6 \mathrm{~Hz}, 1 \mathrm{H}\right), 7.41(\mathrm{t}$, $\left.{ }^{3} J_{\mathrm{HH}}=7.1 \mathrm{~Hz}, 1 \mathrm{H}\right), 7.34\left(\mathrm{~d},{ }^{3} J_{\mathrm{HH}}=7.7 \mathrm{~Hz}, 1 \mathrm{H}\right), 6.84(\mathrm{~s}, 1 \mathrm{H}), 6.82(\mathrm{~s}, 2 \mathrm{H}), 2.92(\mathrm{~s}, 2 \mathrm{H})$, $2.26(\mathrm{~s}, 6 \mathrm{H}), 2.26\left(\mathrm{td}, J_{\mathrm{HH}}=12.9 \mathrm{~Hz}\right.$ and $\left.{ }^{3} J_{\mathrm{HH}}=4.2 \mathrm{~Hz}, 1 \mathrm{H}\right), 2.13\left(\mathrm{td}, J_{\mathrm{HH}}=12.9 \mathrm{~Hz}\right.$ and $\left.{ }^{3} J_{\mathrm{HH}}=4.4 \mathrm{~Hz}, 1 \mathrm{H}\right), 1.35-1.26(\mathrm{~m}, 2 \mathrm{H}), 1.32-1.13(\mathrm{~m}, 1 \mathrm{H}), 0.89-0.81(\mathrm{~m}, 1 \mathrm{H}), 0.84\left(\mathrm{t},{ }^{3} J_{\mathrm{HH}}\right.$ $=7.3 \mathrm{~Hz}, 3 \mathrm{H}) .{ }^{13} \mathrm{C} \mathrm{NMR}\left(\mathrm{CDCl}_{3}\right): \delta 206.4,161.3,147.2,138.2,136.9,135.1,128.2,127.8$, 126.4, 124.5, 123.5, 53.1, 49.9, 40.0, 27.3, 23.4, 21.7, 14.1. HRMS (ESI) calcd for $\mathrm{C}_{21} \mathrm{H}_{24} \mathrm{ONa}\left(\mathrm{M}+\mathrm{Na}^{+}\right) 315.1719$, found 315.1721. 


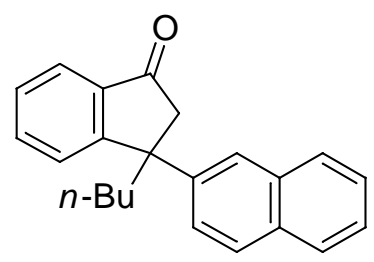

Entry 5. Pale yellow oil. 77\% yield.

2k

${ }^{1} \mathrm{H}$ NMR $\left(\mathrm{CDCl}_{3}\right): \delta 7.83\left(\mathrm{~d},{ }^{3} J_{\mathrm{HH}}=7.7 \mathrm{~Hz}, 1 \mathrm{H}\right), 7.80-7.73(\mathrm{~m}, 4 \mathrm{H}), 7.62\left(\mathrm{t},{ }^{3} J_{\mathrm{HH}}=6.9\right.$ $\mathrm{Hz}, 1 \mathrm{H}), 7.49-7.43(\mathrm{~m}, 3 \mathrm{H}), 7.36\left(\mathrm{~d},{ }^{3} J_{\mathrm{HH}}=7.7 \mathrm{~Hz}, 1 \mathrm{H}\right), 7.27-7.24(\mathrm{~m}, 1 \mathrm{H}), 3.02(\mathrm{~s}, 2 \mathrm{H})$, $2.40\left(\mathrm{td}, J_{\mathrm{HH}}=12.9 \mathrm{~Hz}\right.$ and $\left.{ }^{3} J_{\mathrm{HH}}=4.3 \mathrm{~Hz}, 1 \mathrm{H}\right), 2.27\left(\mathrm{td}, J_{\mathrm{HH}}=12.9 \mathrm{~Hz}\right.$ and ${ }^{3} J_{\mathrm{HH}}=4.3$ $\mathrm{Hz}, 1 \mathrm{H}), 1.40-1.31(\mathrm{~m}, 2 \mathrm{H}), 1.30-1.21(\mathrm{~m}, 1 \mathrm{H}), 0.96-0.87(\mathrm{~m}, 1 \mathrm{H}), 0.87\left(\mathrm{t},{ }^{3} J_{\mathrm{HH}}=7.3 \mathrm{~Hz}\right.$, $3 \mathrm{H}) .{ }^{13} \mathrm{C}$ NMR $\left(\mathrm{CDCl}_{3}\right): \delta$ 206.0, 161.0, 144.4, 137.1, 135.2, 133.4, 132.1, 128.7, 128.2, 128.1, 127.6, 126.5, 126.4, 126.1, 125.6, 124.7, 123.7, 52.9, 50.2, 39.9, 27.3, 23.4, 14.2. HRMS (ESI) calcd for $\mathrm{C}_{23} \mathrm{H}_{22} \mathrm{ONa}\left(\mathrm{M}+\mathrm{Na}^{+}\right) 337.1563$, found 337.1562 .

Entry 6. Pale yellow oil. 53\% yield.

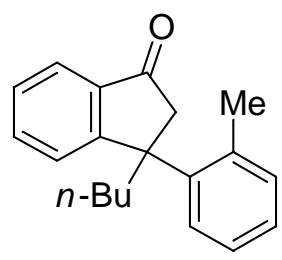

2)

${ }^{1} \mathrm{H} \mathrm{NMR}\left(\mathrm{CDCl}_{3}\right): \delta 7.79\left(\mathrm{~d},{ }^{3} J_{\mathrm{HH}}=7.6 \mathrm{~Hz}, 1 \mathrm{H}\right), 7.59\left(\mathrm{t}^{3}{ }^{3} \mathrm{~J}_{\mathrm{HH}}=7.3 \mathrm{~Hz}, 1 \mathrm{H}\right), 7.45-7.41$ $(\mathrm{m}, 2 \mathrm{H}), 7.26\left(\mathrm{~d},{ }^{3} \mathrm{~J}_{\mathrm{HH}}=7.5 \mathrm{~Hz}, 1 \mathrm{H}\right), 7.20-7.15(\mathrm{~m}, 2 \mathrm{H}), 7.12-7.10(\mathrm{~m}, 1 \mathrm{H}), 3.01\left(\mathrm{~d},{ }^{2} J_{\mathrm{HH}}\right.$ $=19.3 \mathrm{~Hz}, 1 \mathrm{H}), 2.92\left(\mathrm{~d},{ }^{2} J_{\mathrm{HH}}=19.3 \mathrm{~Hz}, 1 \mathrm{H}\right), 2.25-2.16(\mathrm{~m}, 2 \mathrm{H}), 1.87(\mathrm{~s}, 3 \mathrm{H}), 1.32-1.23$ $(\mathrm{m}, 2 \mathrm{H}), 1.15-1.06(\mathrm{~m}, 1 \mathrm{H}), 0.81\left(\mathrm{t},{ }^{3} \mathrm{~J}_{\mathrm{HH}}=7.2 \mathrm{~Hz}, 3 \mathrm{H}\right), 0.76-0.68(\mathrm{~m}, 1 \mathrm{H}) .{ }^{13} \mathrm{C}$ NMR $\left(\mathrm{CDCl}_{3}\right): \delta 206.2,161.6,143.8,137.3,136.7,135.0,133.3,127.8,127.2,127.1,126.3,125.9$, 123.7, 51.2, 49.9, 41.3, 27.0, 23.3, 22.0, 14.1. Anal. Calcd for $\mathrm{C}_{20} \mathrm{H}_{22} \mathrm{O}: \mathrm{C}, 86.29 ; \mathrm{H}, 7.97$. Found: C, 86.02; H, 7.92.

\section{Procedure for Equation 2.}

Substrate $4(38.3 \mathrm{mg}, 0.20 \mathrm{mmol})$ was added to a solution of $\left[\mathrm{RhCl}\left(\mathrm{C}_{2} \mathrm{H}_{4}\right)_{2}\right]_{2}(3.9$ $\mathrm{mg}, 20 \mu \mathrm{mol} \mathrm{Rh})$ and dppf (12.2 mg, $22 \mu \mathrm{mol})$ in 1,2-dichloroethane (10 mL). PhZnCl $(625 \mu \mathrm{L}, 0.30 \mathrm{mmol} ; 0.48 \mathrm{M}$ in THF) was then added to it and the resulting solution was stirred for $17 \mathrm{~h}$ at $25^{\circ} \mathrm{C}$. The reaction was quenched with water $(30 \mu \mathrm{L})$ and passed through a pad of silica gel with $\mathrm{Et}_{2} \mathrm{O}$. The solvent was removed under vacuum and the residue was purified by silica gel preparative TLC with $\mathrm{Et}_{2} \mathrm{O} /$ hexane $=1 / 5$ to affordindanone 5 as a colorless oil $(41.8 \mathrm{mg}, 0.16 \mathrm{mmol} ; 78 \%$ yield).

${ }^{1} \mathrm{H} \mathrm{NMR}\left(\mathrm{CDCl}_{3}\right): \delta$ 7.31-7.27 (m, 2H), 7.23-7.18 (m, 3H), 2.95 (bs, 0.73H), 2.92 (bs, $0.27 \mathrm{H}), 2.27\left(\mathrm{ddd},{ }^{2} J_{\mathrm{HH}}=13.6 \mathrm{~Hz}\right.$ and ${ }^{3} J_{\mathrm{HH}}=12.5$ and $\left.4.3 \mathrm{~Hz}, 1 \mathrm{H}\right), 2.15\left(\mathrm{ddd},{ }^{2} J_{\mathrm{HH}}=\right.$ $13.4 \mathrm{~Hz}$ and ${ }^{3} J_{\mathrm{HH}}=12.3$ and $\left.4.2 \mathrm{~Hz}, 1 \mathrm{H}\right), 1.34-1.26(\mathrm{~m}, 2 \mathrm{H}), 1.25-1.16(\mathrm{~m}, 1 \mathrm{H}), 0.92-$ $0.85(\mathrm{~m}, 1 \mathrm{H}), 0.84\left(\mathrm{t},{ }^{3} \mathrm{~J}_{\mathrm{HH}}=7.3 \mathrm{~Hz}, 3 \mathrm{H}\right) .{ }^{13} \mathrm{C} \mathrm{NMR}\left(\mathrm{CDCl}_{3}\right): \delta 206.1,160.9,147.18$ (minor) and 147.16 (major), 136.9, $134.6\left(\mathrm{t},{ }^{1} J_{\mathrm{CD}}=25.3 \mathrm{~Hz}\right), 128.7,127.4\left(\mathrm{t},{ }^{1} J_{\mathrm{CD}}=25.3\right.$ $\mathrm{Hz}), 126.6,126.5,125.9\left(\mathrm{t},{ }^{1} J_{\mathrm{CD}}=24.8 \mathrm{~Hz}\right), 123.1\left(\mathrm{t},{ }^{1} J_{\mathrm{CD}}=24.2 \mathrm{~Hz}\right), 52.8\left(\mathrm{t},{ }^{1} J_{\mathrm{CD}}=20.5\right.$ $\mathrm{Hz}$; minor) and $52.7\left(\mathrm{t},{ }^{1} J_{\mathrm{CD}}=20.7 \mathrm{~Hz}\right.$; major), 50.0, 39.99 (major) and 39.98 (minor), 
27.3, 23.3, 14.1. ${ }^{2} \mathrm{H}$ NMR $\left(\mathrm{CHCl}_{3}\right): \delta 7.83(\mathrm{bs}, 1 \mathrm{H}), 7.65(\mathrm{bs}, 1 \mathrm{H}), 7.46(\mathrm{bs}, 1 \mathrm{H}), 7.38$ (bs, $1 \mathrm{H}), 2.92$ (bs, 1H).

\section{Procedure for Equation 3.}

1-Phenyl-2-heptyn-1-one $\mathbf{1 a}(37.3 \mathrm{mg}, 0.20 \mathrm{mmol})$ was added to a solution of $\left[\mathrm{RhCl}\left(\mathrm{C}_{2} \mathrm{H}_{4}\right)_{2}\right]_{2}(3.9 \mathrm{mg}, 20 \mu \mathrm{mol} \mathrm{Rh})$ and dppf $(12.2 \mathrm{mg}, 22 \mu \mathrm{mol})$ in 1,2dichloroethane $(12 \mathrm{~mL})$. PhZnCl $(625 \mu \mathrm{L}, 0.30 \mathrm{mmol} ; 0.48 \mathrm{M}$ in THF) was then added to it and the resulting solution was stirred for $17 \mathrm{~h}$ at $25^{\circ} \mathrm{C}$. The reaction mixture was cooled to $0{ }^{\circ} \mathrm{C}$ and allyl bromide $(51.9 \mu \mathrm{L}, 0.60 \mathrm{mmol})$ was added dropwise to it. After stirring for $6 \mathrm{~h}$ at $25^{\circ} \mathrm{C}$, the mixture was directly passed through a pad of silica gel with $\mathrm{Et}_{2} \mathrm{O}$ and the solvent was removed under vacuum. The residue was purified by silica gel preparative TLC with $\mathrm{Et}_{2} \mathrm{O} /$ hexane $=1 / 8$ to afford allylated indanone 6 as a pale yellow oil $(36.4 \mathrm{mg}, 0.12 \mathrm{mmol} ; 60 \%$ yield; d.r. $=2.1 / 1)$. HRMS (ESI) calcd for $\mathrm{C}_{22} \mathrm{H}_{24} \mathrm{ONa}\left(\mathrm{M}+\mathrm{Na}^{+}\right) 327.1719$, found 327.1720 .

Major diastereomer: ${ }^{1} \mathrm{H}$ NMR $\left(\mathrm{CDCl}_{3}\right): \delta 7.82\left(\mathrm{~d},{ }^{3} \mathrm{~J}_{\mathrm{HH}}=7.5 \mathrm{~Hz}, 1 \mathrm{H}\right), 7.59\left(\mathrm{t},{ }^{3} \mathrm{~J}_{\mathrm{HH}}=\right.$ $7.8 \mathrm{~Hz}, 1 \mathrm{H}), 7.43\left(\mathrm{t},{ }^{3} \mathrm{~J}_{\mathrm{HH}}=7.2 \mathrm{~Hz}, 1 \mathrm{H}\right), 7.32-7.21(\mathrm{~m}, 3 \mathrm{H}), 7.19-7.15(\mathrm{~m}, 1 \mathrm{H}), 6.96(\mathrm{~d}$, $\left.{ }^{3} J_{\mathrm{HH}}=7.2 \mathrm{~Hz}, 2 \mathrm{H}\right), 5.78-5.66(\mathrm{~m}, 1 \mathrm{H}), 4.88\left(\mathrm{~d},{ }^{3} J_{\mathrm{HH}}=9.6 \mathrm{~Hz}, 1 \mathrm{H}\right), 4.75\left(\mathrm{dd},{ }^{3} J_{\mathrm{HH}}=16.9\right.$ $\mathrm{Hz}$ and $\left.{ }^{4} J_{\mathrm{HH}}=1.5 \mathrm{~Hz}, 1 \mathrm{H}\right), 2.82\left(\mathrm{dd},{ }^{3} J_{\mathrm{HH}}=7.8\right.$ and $\left.6.2 \mathrm{~Hz}, 1 \mathrm{H}\right), 2.36\left(\mathrm{dt}^{2} J_{\mathrm{HH}}=13.7\right.$ $\mathrm{Hz}$ and $\left.{ }^{3} \mathrm{~J}_{\mathrm{HH}}=4.7 \mathrm{~Hz}, 1 \mathrm{H}\right), 2.35-2.28(\mathrm{~m}, 2 \mathrm{H}), 1.67-1.60(\mathrm{~m}, 1 \mathrm{H}), 1.40-1.32(\mathrm{~m}, 1 \mathrm{H})$, 1.27-1.17 (m, 2H), 1.06-0.96 (m, 1H), $0.87\left(\mathrm{t}^{3} \mathrm{~J}_{\mathrm{HH}}=7.3 \mathrm{~Hz}, 3 \mathrm{H}\right) .{ }^{13} \mathrm{C} \mathrm{NMR}\left(\mathrm{CDCl}_{3}\right)$ : $\delta$ 207.3 159.3, 144.1, 136.78, 136.77, 135.1, 128.3, 128.2, 127.8, 126.7, 126.1, 123.5, 116.1, $57.8,54.0,38.0,32.9,26.9,23.5,14.2$.

Minor diastereomer: ${ }^{1} \mathrm{H}$ NMR $\left(\mathrm{CDCl}_{3}\right): \delta 7.81\left(\mathrm{~d},{ }^{3} J_{\mathrm{HH}}=6.2 \mathrm{~Hz}, 1 \mathrm{H}\right), 7.57\left(\mathrm{t},{ }^{3} J_{\mathrm{HH}}=\right.$ $7.7 \mathrm{~Hz}, 1 \mathrm{H}), 7.43\left(\mathrm{t},{ }^{3} \mathrm{~J}_{\mathrm{HH}}=7.2 \mathrm{~Hz}, 1 \mathrm{H}\right), 7.32-7.21(\mathrm{~m}, 5 \mathrm{H}), 7.19-7.15(\mathrm{~m}, 1 \mathrm{H}), 5.78-5.66$ $(\mathrm{m}, 1 \mathrm{H}), 5.03\left(\mathrm{dd},{ }^{3} \mathrm{~J}_{\mathrm{HH}}=16.4 \mathrm{~Hz}\right.$ and $\left.{ }^{4} \mathrm{~J}_{\mathrm{HH}}=1.4 \mathrm{~Hz}, 1 \mathrm{H}\right), 4.90\left(\mathrm{~d},{ }^{3} \mathrm{~J}_{\mathrm{HH}}=8.5 \mathrm{~Hz}, 1 \mathrm{H}\right)$, 2.84-2.79 (m, 2H), 2.33-2.26 (m, 1H), $2.22\left(\mathrm{dt}^{2} \mathrm{~J}_{\mathrm{HH}}=13.1 \mathrm{~Hz}\right.$ and $\left.{ }^{3} J_{\mathrm{HH}}=4.4 \mathrm{~Hz}, 1 \mathrm{H}\right)$, $1.90\left(\mathrm{dt}^{2} \mathrm{~J}_{\mathrm{HH}}=12.9 \mathrm{~Hz}\right.$ and $\left.{ }^{3} \mathrm{~J}_{\mathrm{HH}}=4.1 \mathrm{~Hz}, 1 \mathrm{H}\right), 1.67-1.60(\mathrm{~m}, 1 \mathrm{H}), 1.40-1.32(\mathrm{~m}, 2 \mathrm{H})$, $0.80\left(\mathrm{t},{ }^{3} \mathrm{~J}_{\mathrm{HH}}=7.3 \mathrm{~Hz}, 3 \mathrm{H}\right), 0.75-0.66(\mathrm{~m}, 1 \mathrm{H}) .{ }^{13} \mathrm{C} \mathrm{NMR}\left(\mathrm{CDCl}_{3}\right): \delta 206.1,160.0,146.3$, $137.3,136.5,134.4,128.5,128.0,127.9,126.7,126.6,123.3,115.9,63.7,53.8,35.7,30.0$, $27.7,23.3,14.1$.

\section{Procedure for Equation 4.}

1-Phenyl-2-heptyn-1-one $1 \mathrm{a}(18.6 \mathrm{mg}, 0.10 \mathrm{mmol})$ was added to a solution of $\left[\mathrm{RhCl}\left(\mathrm{C}_{2} \mathrm{H}_{4}\right)_{2}\right]_{2}(1.9 \mathrm{mg}, 9.8 \mu \mathrm{mol} \mathrm{Rh})$ and ligand $7(6.6 \mathrm{mg}, 11 \mu \mathrm{mol})$ in $1,2-$ dichloroethane $(6 \mathrm{~mL})$. $\mathrm{PhZnCl}(313 \mu \mathrm{L}, 0.15 \mathrm{mmol} ; 0.48 \mathrm{M}$ in THF) was then added to it and the resulting solution was stirred for $13 \mathrm{~h}$ at $25{ }^{\circ} \mathrm{C}$. The reaction was quenched with water $(30 \mu \mathrm{L})$ and passed through a pad of silica gel with $\mathrm{Et}_{2} \mathrm{O}$. The solvent was removed under vacuum and the residue was purified by silica gel preparative TLC with $\mathrm{Et}_{2} \mathrm{O} /$ hexane $=1 / 5$ to afford indanone $2 \mathbf{a}$ as a pale yellow oil (17.1 mg, $64.7 \mu \mathrm{mol} ; 65 \%$ yield). The ee was determined using a Daicel Chiralcel OJ$\mathrm{H}$ column with hexane : 2-propanol $=95: 5$, flow $=0.3 \mathrm{~mL} / \mathrm{min}$. Retention times: $34.6 \mathrm{~min}\left[(+)\right.$-enantiomer], $37.1 \mathrm{~min}\left[(-)\right.$-enantiomer]. $54 \%$ ee. $[\alpha]_{\mathrm{D}}^{20}+8.3(c 0.62$, $\left.\mathrm{CHCl}_{3}\right)$. 


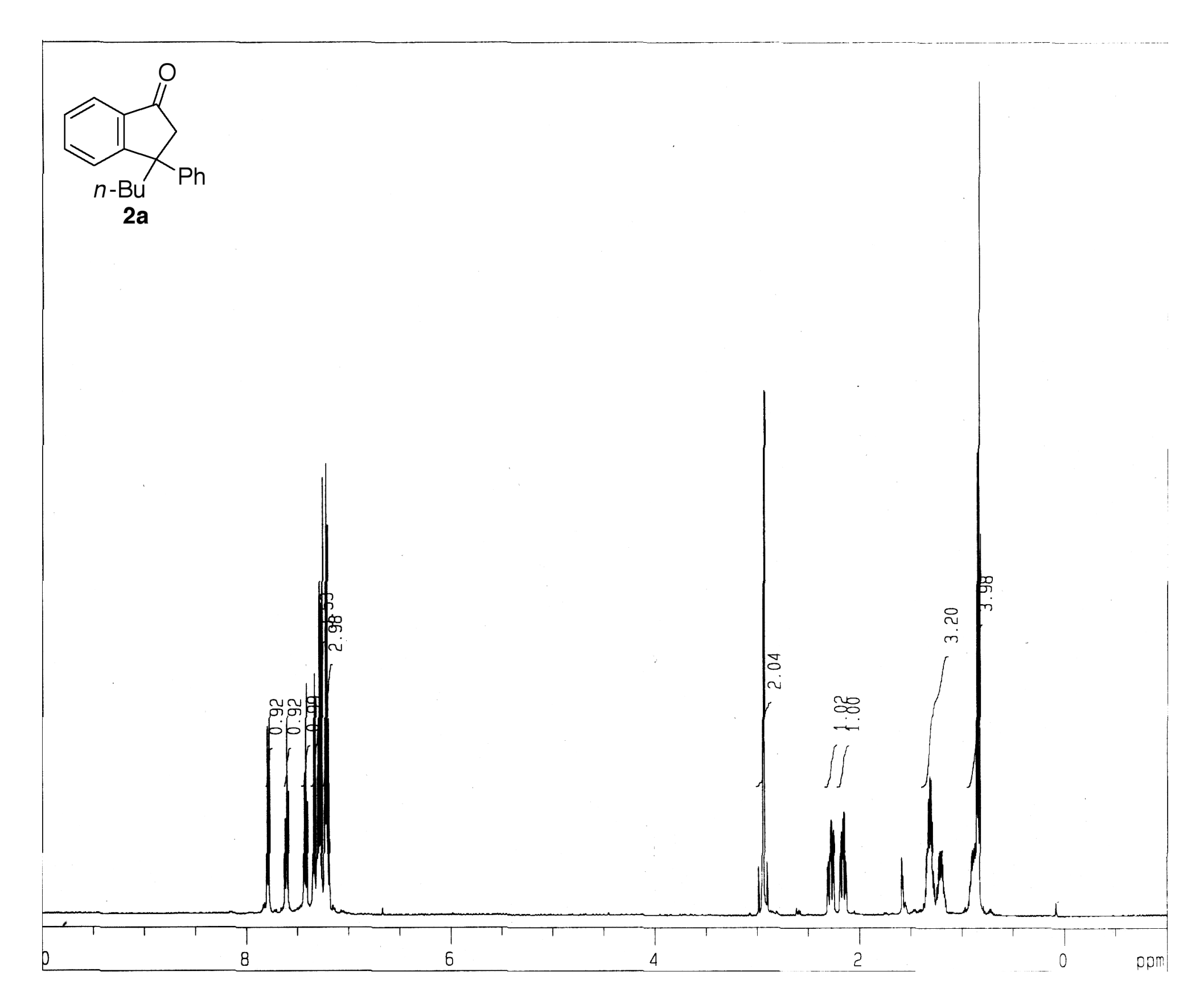




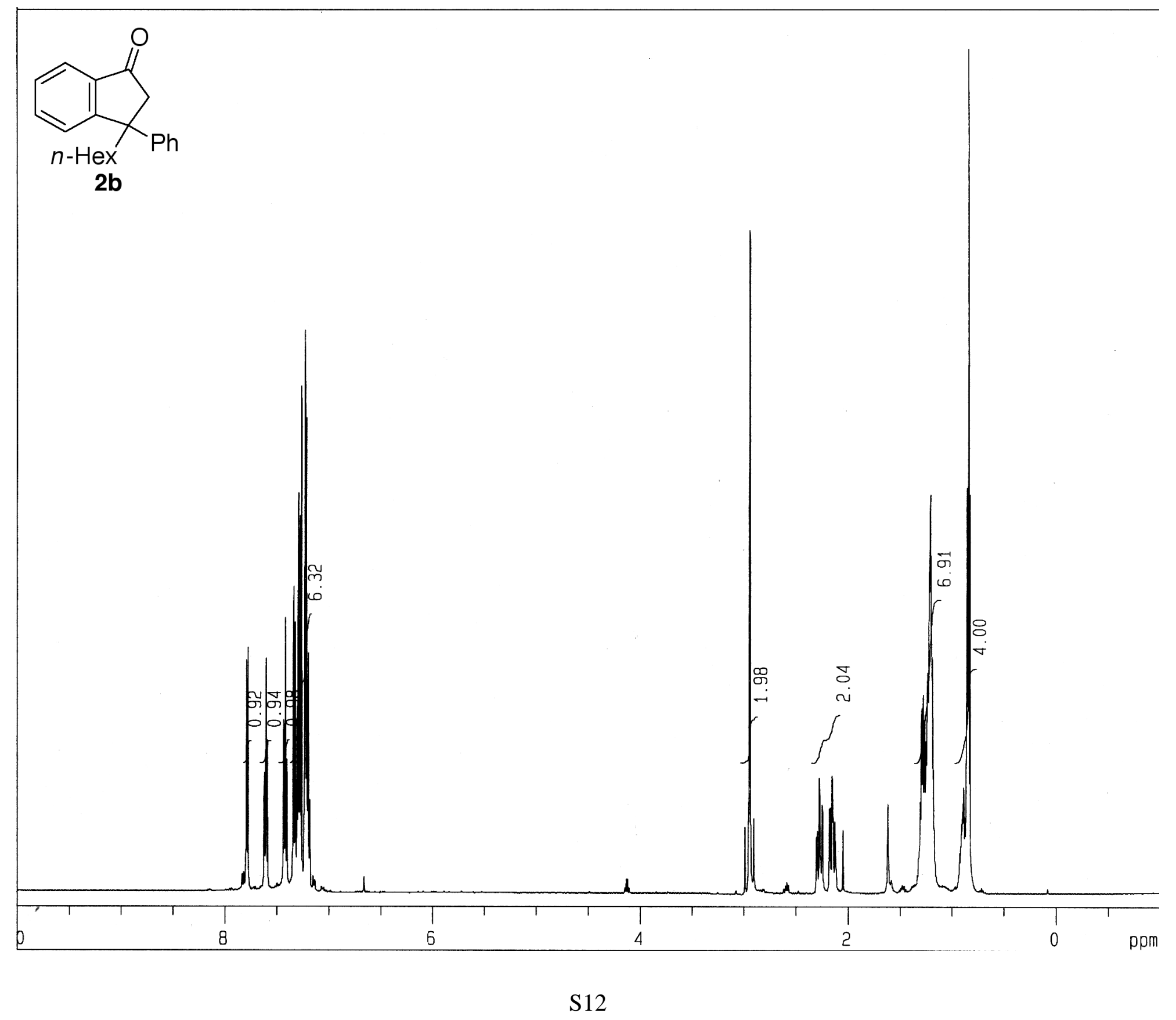




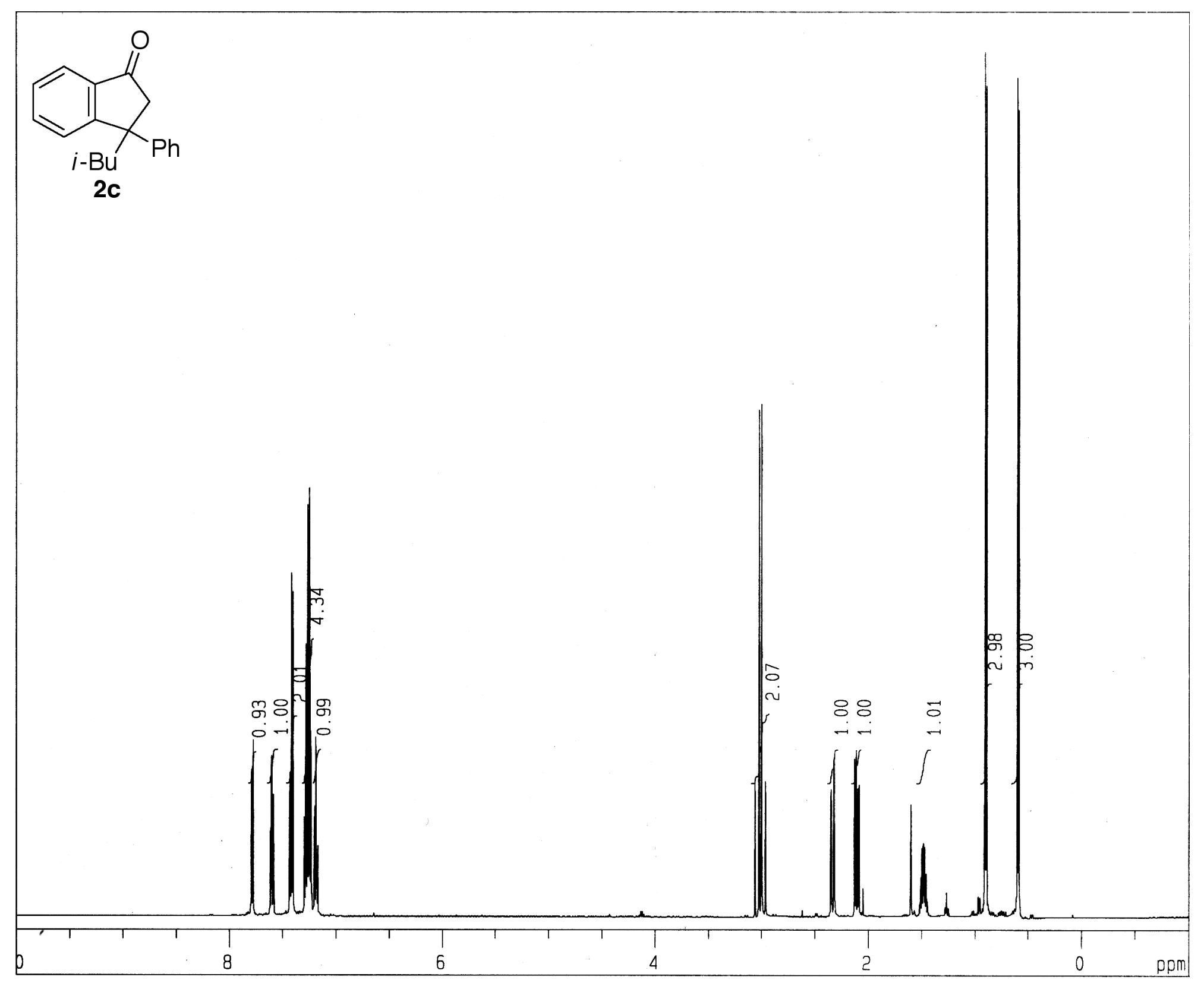

S13 


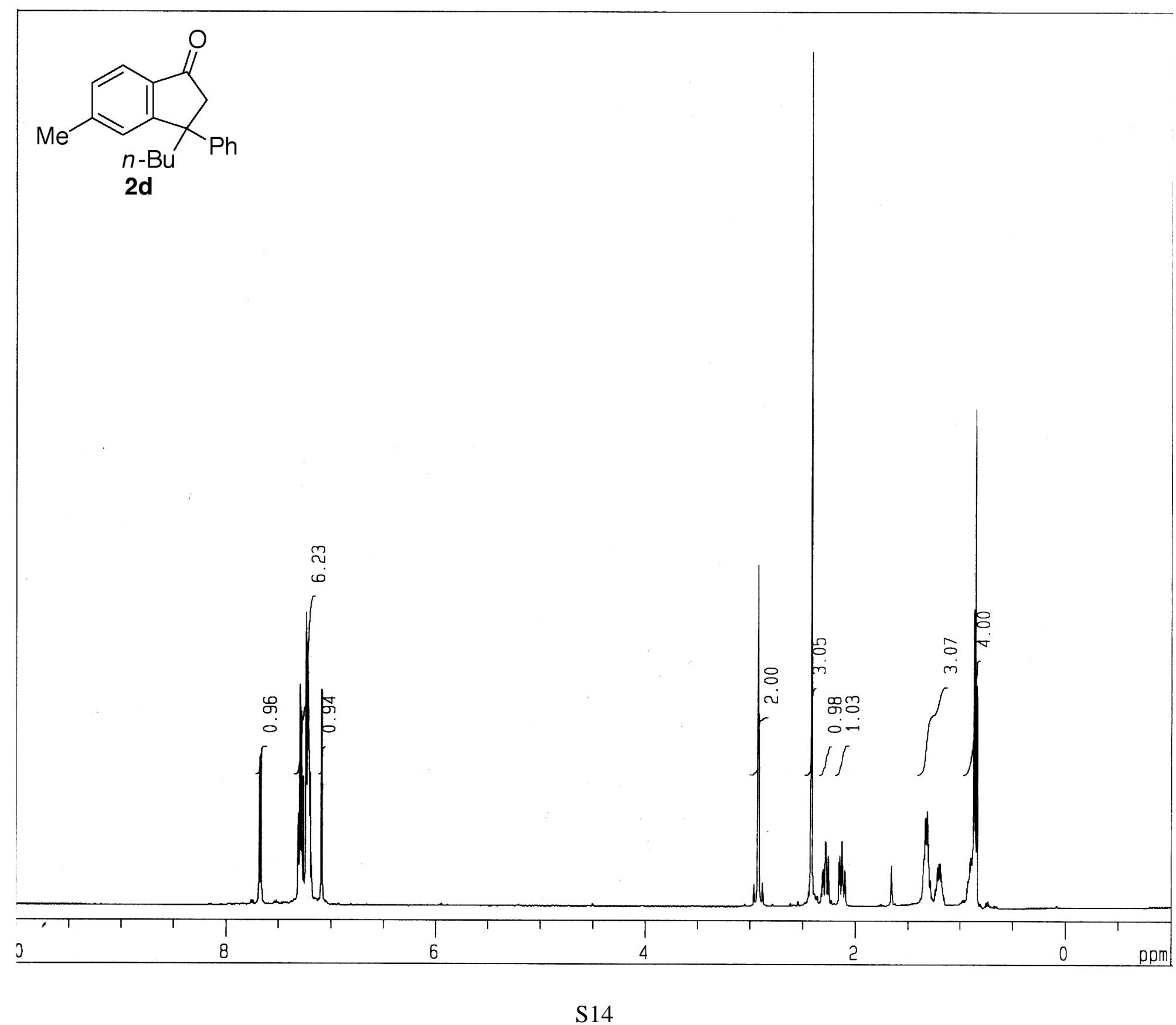




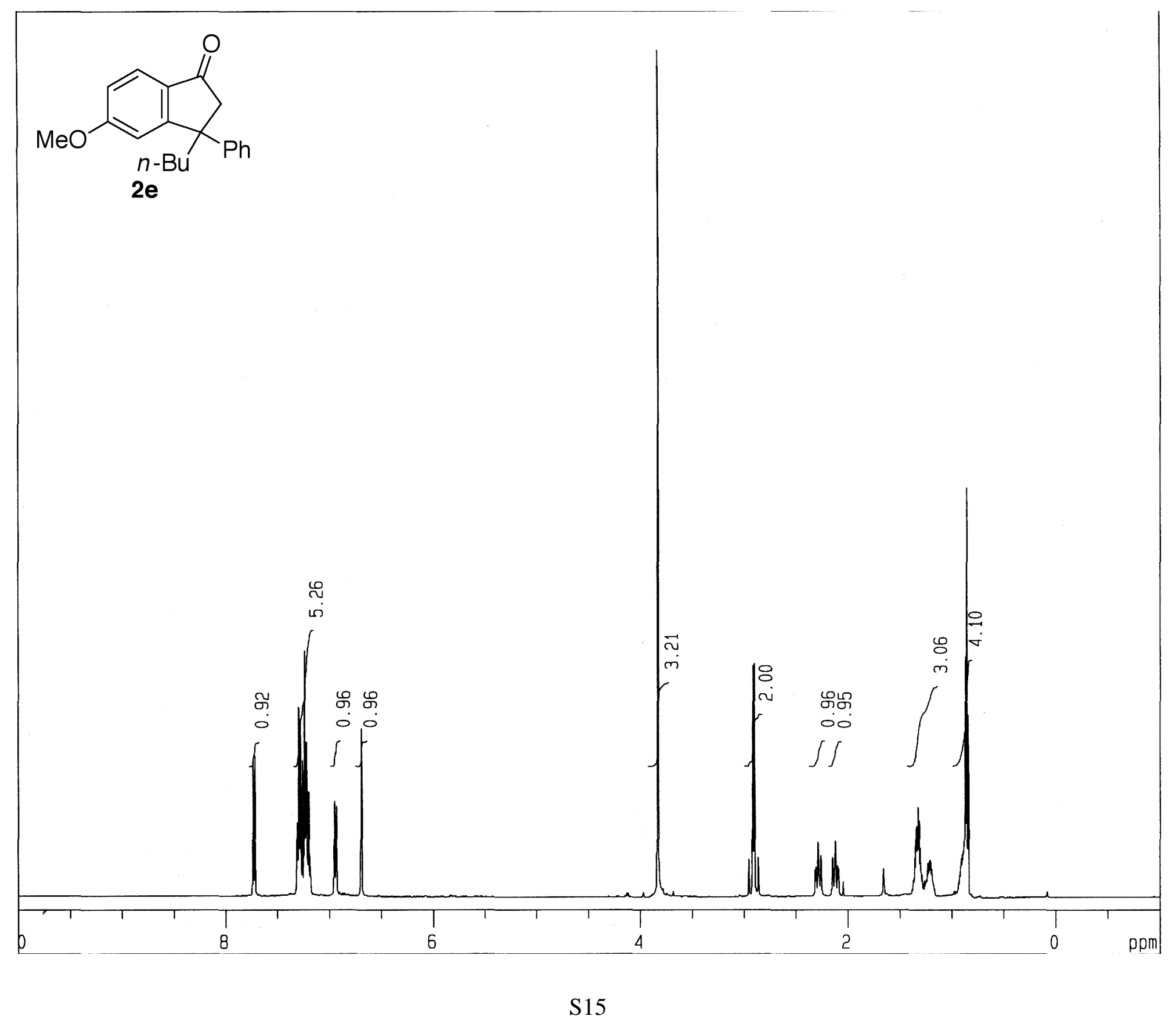




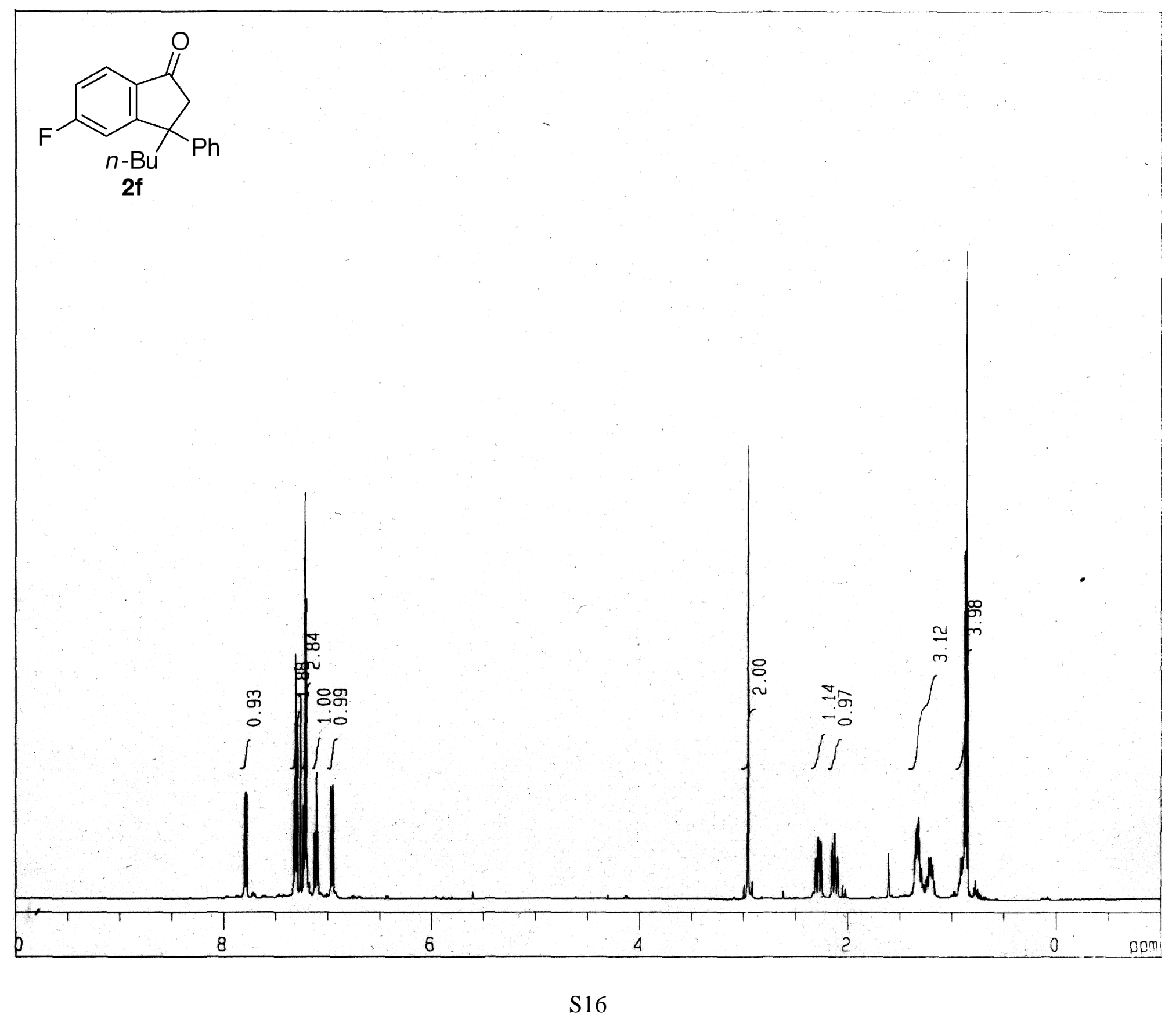




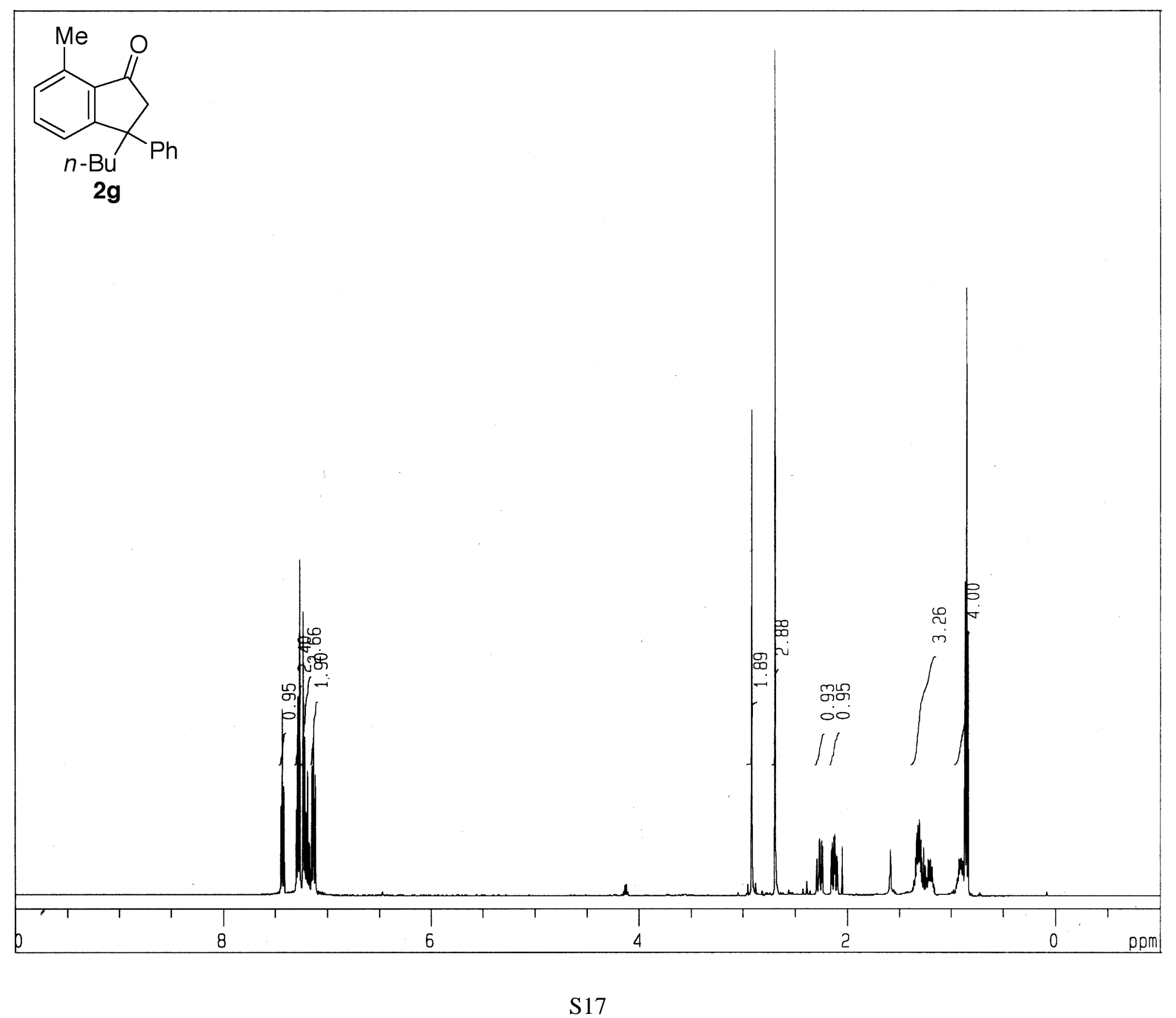




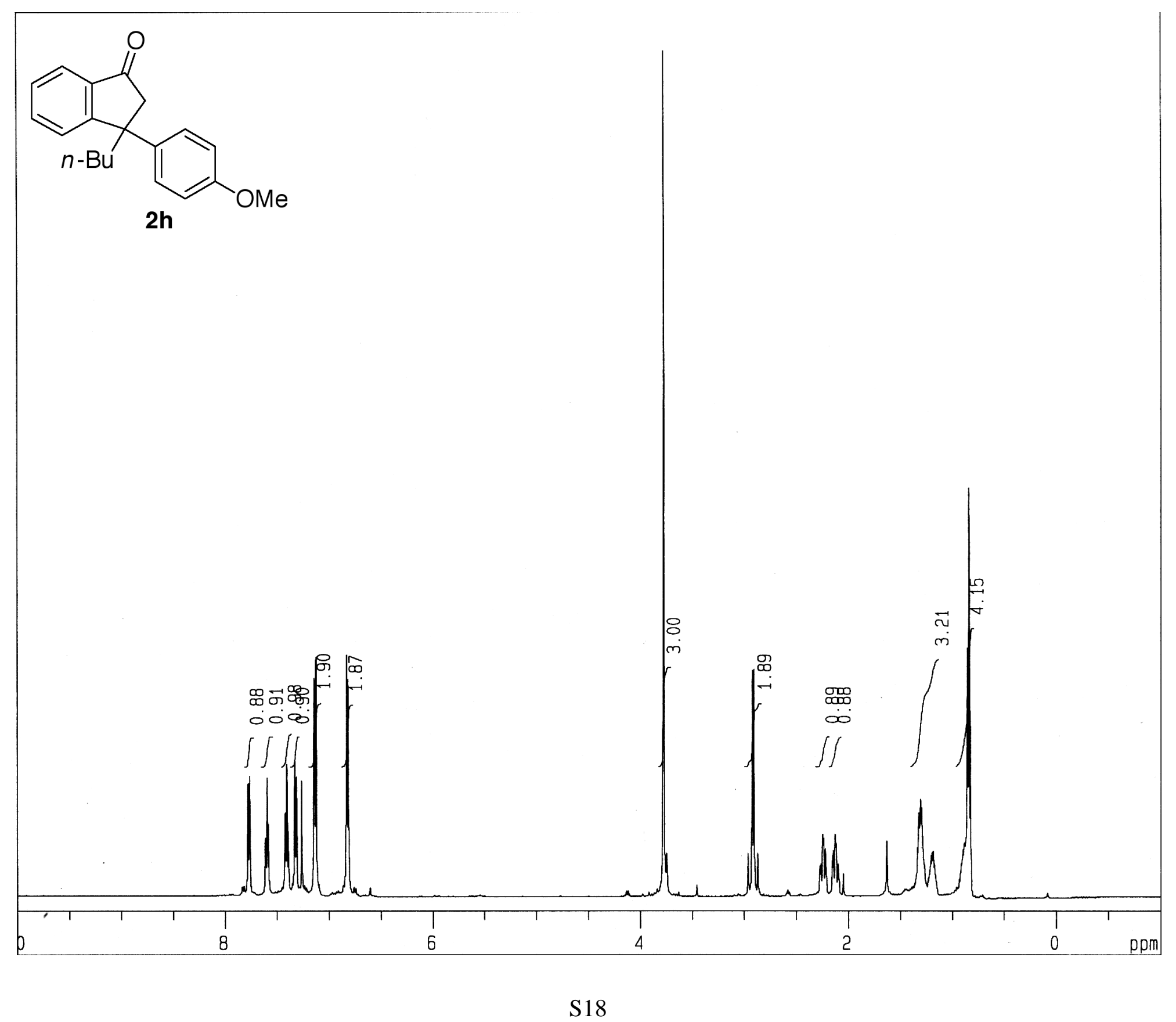




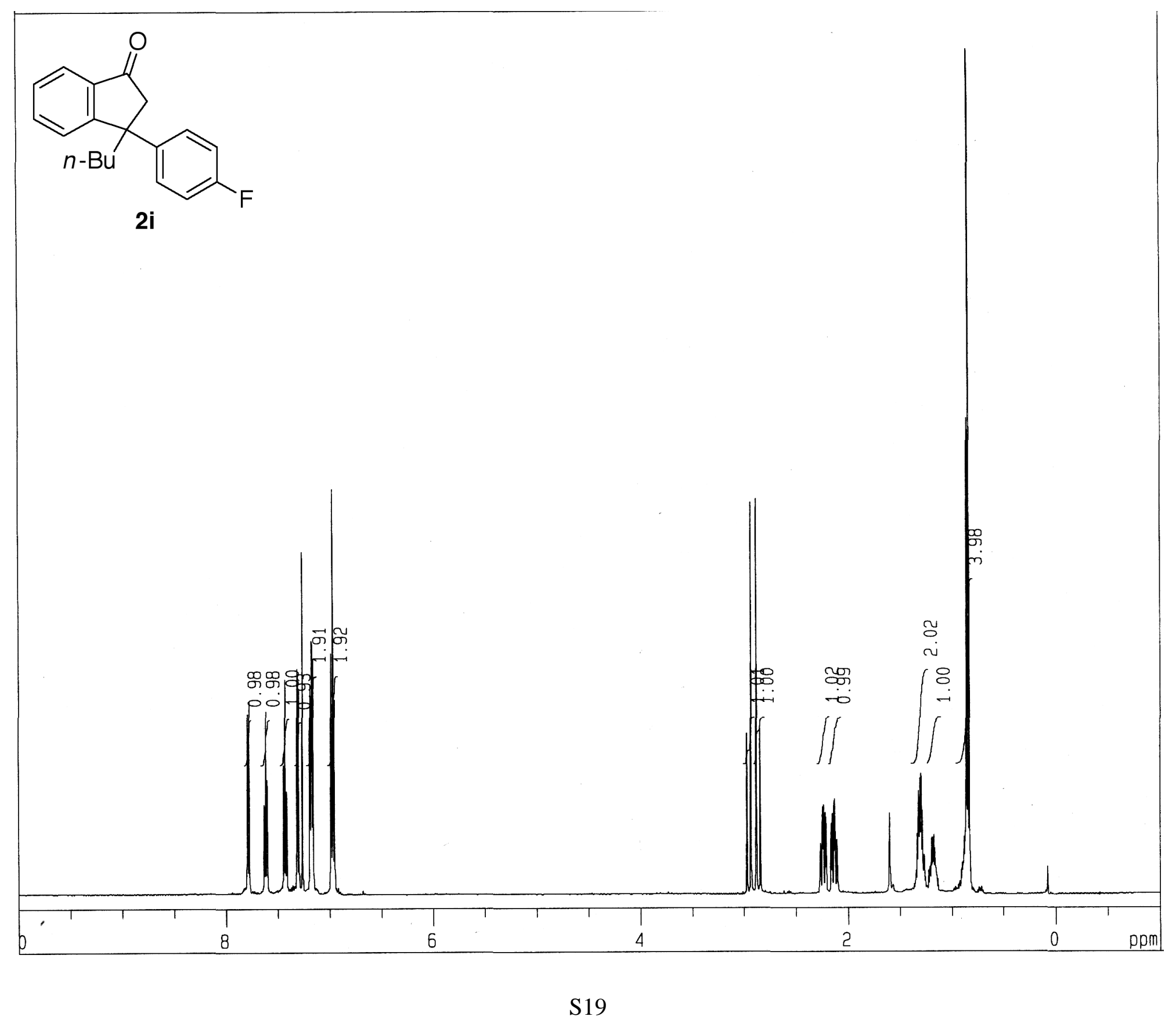




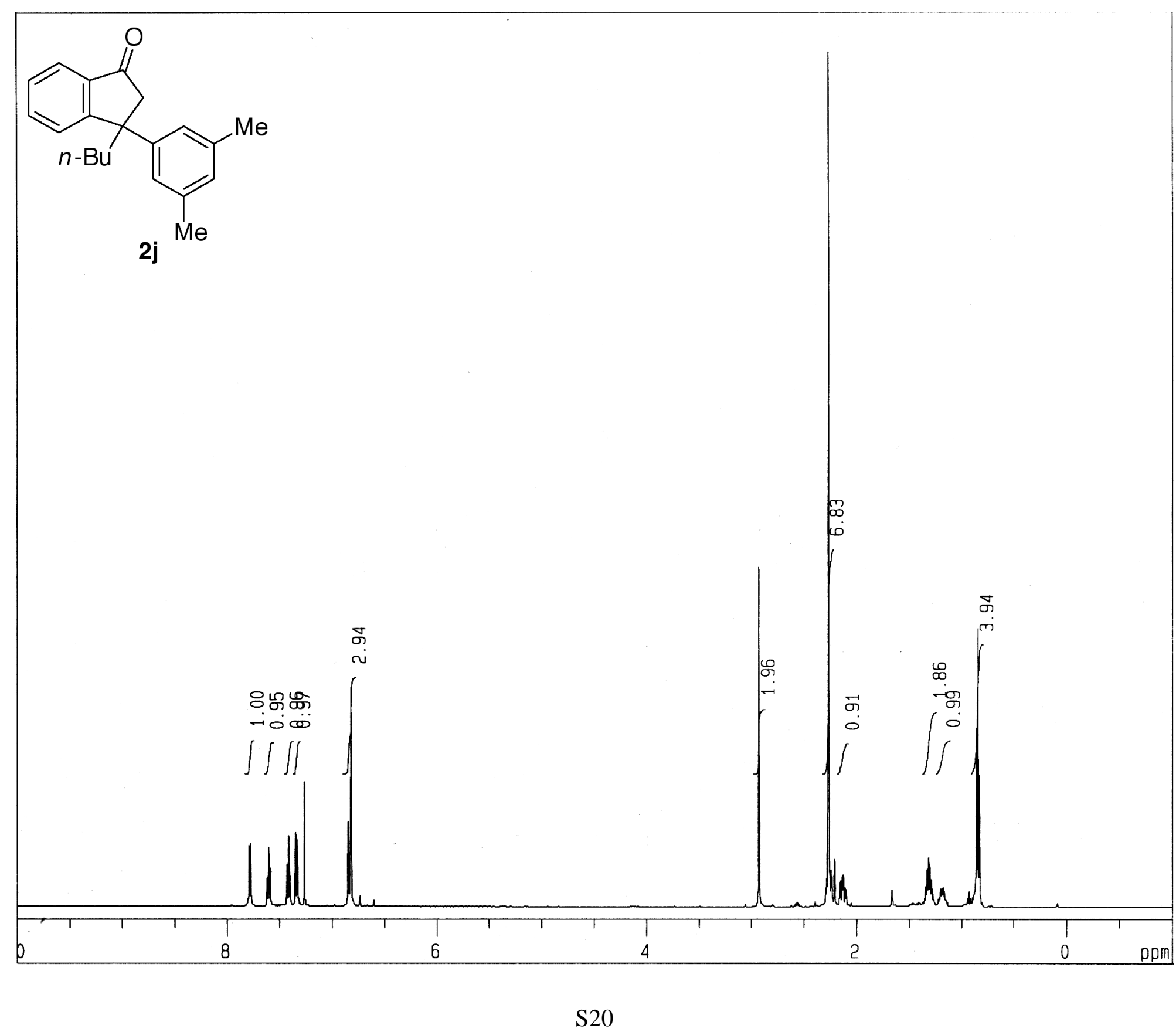




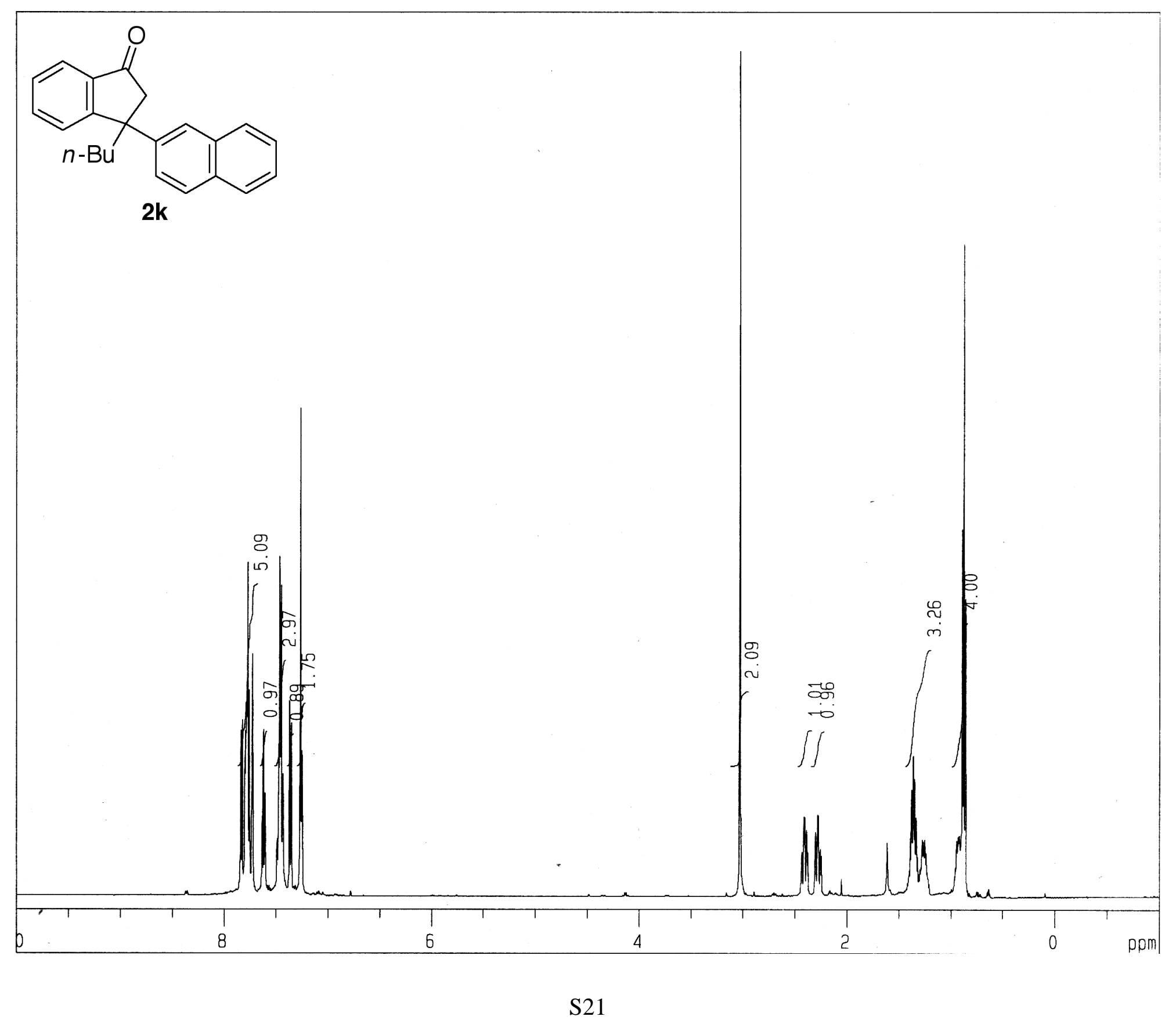




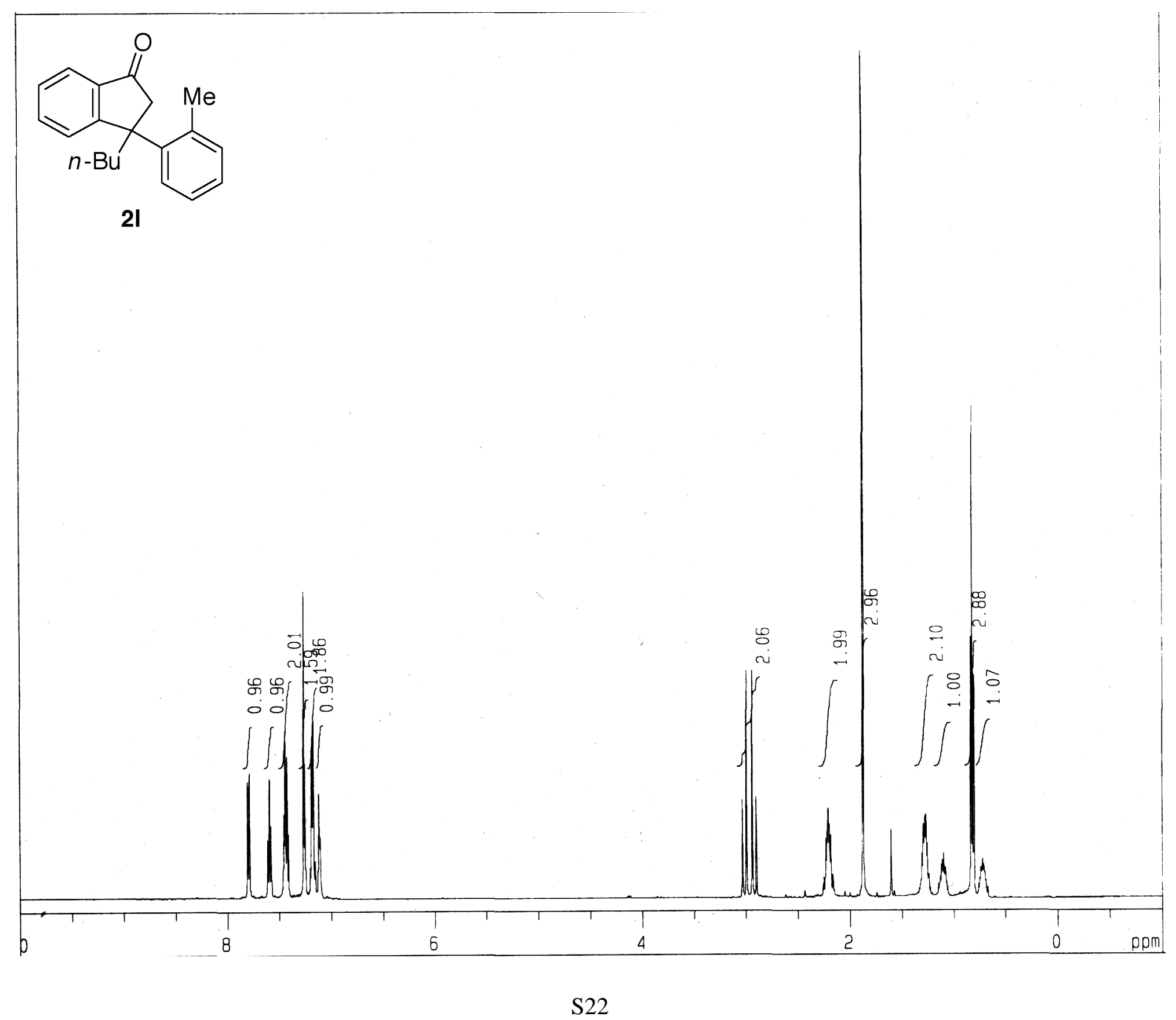




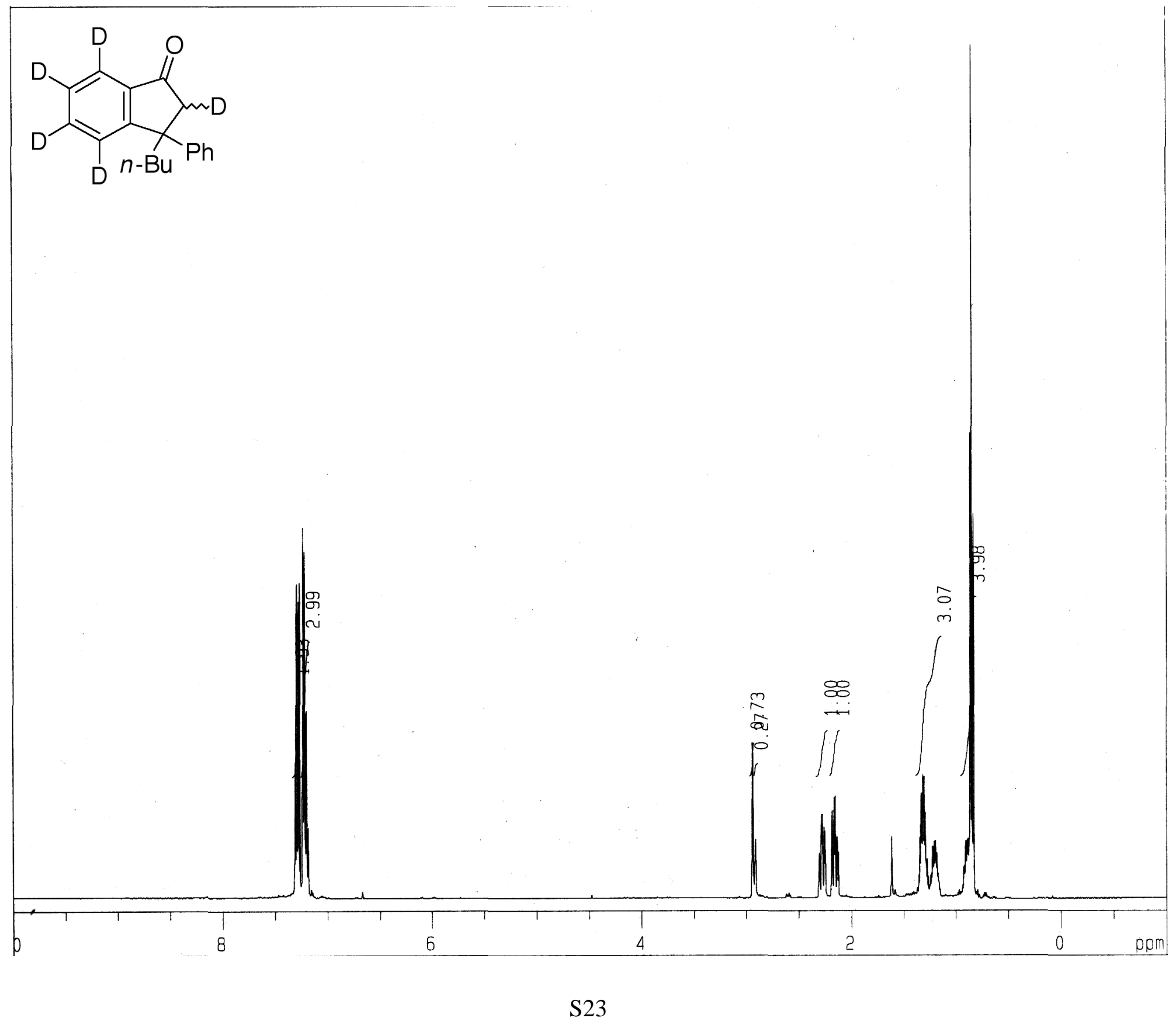




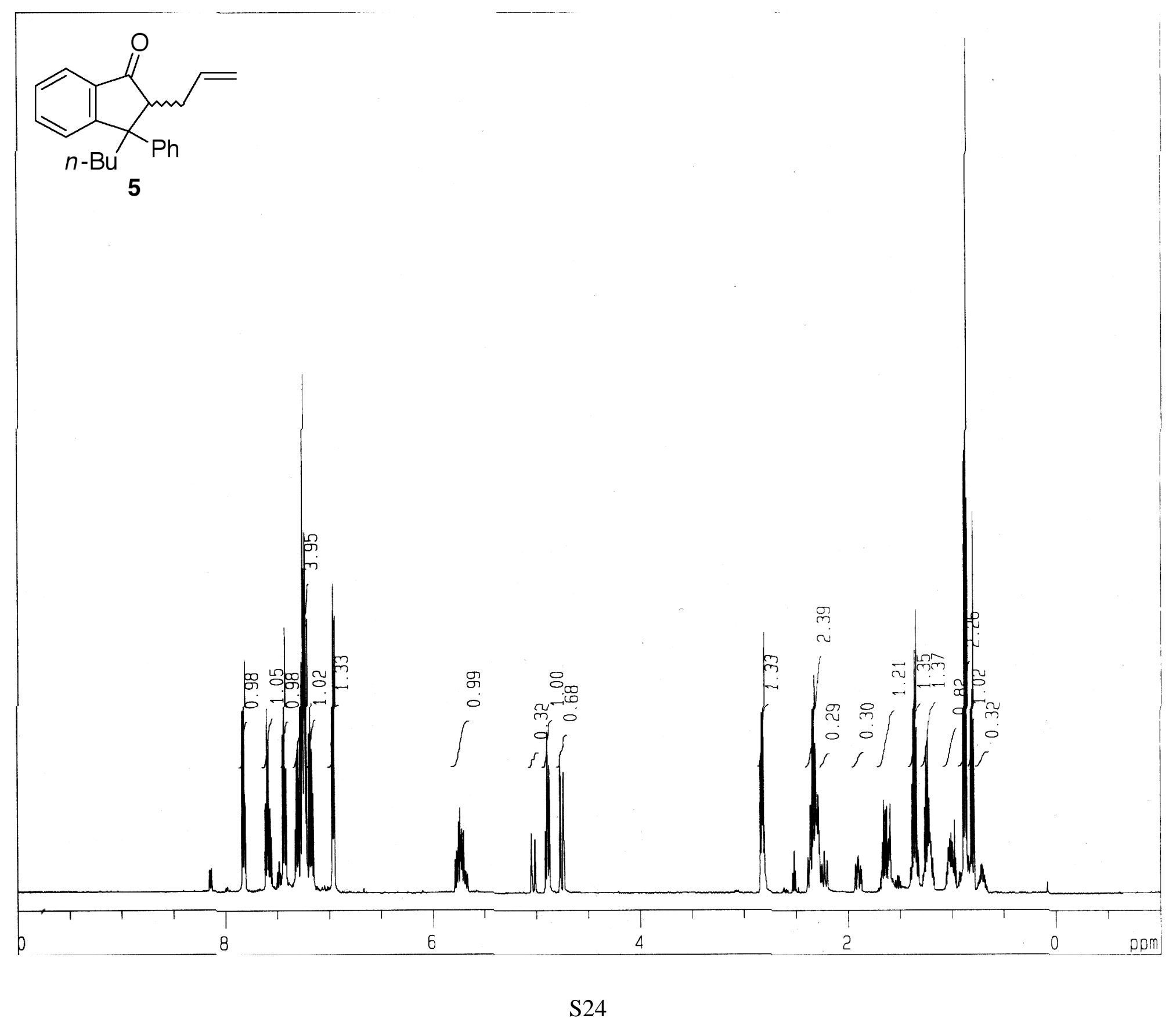

